\title{
Error Rate Analysis of Amplitude-Coherent Detection over Rician Fading Channels with Receiver Diversity
}

Mohammad Al-Jarrah, Member, IEEE, Ki-Hong Park, Member, IEEE, Arafat Al-Dweik, Senior Member, IEEE, and Mohamed-Slim Alouini, Fellow, IEEE

\begin{abstract}
Amplitude-coherent (AC) detection is an efficient technique that can simplify the receiver design while providing reliable symbol error rate (SER). Therefore, this work considers AC detector design and SER analysis using M-ary amplitude shift keying (MASK) modulation with receiver diversity over Rician fading channels. More specifically, we derive the optimum, near-optimum and a suboptimum AC detectors and compare their SER with the coherent, phase-coherent, noncoherent and the heuristic AC detectors. Moreover, the analytical and asymptotic SER at high signal-to-noise ratios (SNRs) are derived for the heuristic detector using single and multiple receiving antennas. The obtained analytical and simulation results show that the SER of the AC and coherent MASK detectors are comparable, particularly for high values of the Rician K-factor, and small number of receiving antennas. In most of the considered scenarios, the heuristic AC detector outperforms the optimum noncoherent detector significantly, except for the binary ASK case at low SNRs. Moreover, the obtained results show that the heuristic AC detector is immune to phase noise, and thus, it outperforms the coherent detector in scenarios where the system is subject to considerable phase noise.
\end{abstract}

Index Terms-Optical wireless communications (OWC), free space optics (FSO), noncoherent, semi-coherent, amplitude-coherent, Rician fading, receiver diversity, phase noise.

M. Al-Jarrah and A. Al-Dweik are with the Center for Cyber Physical Systems, Khalifa University, Abu Dhabi, UAE. (E-mail: \{mohammad.aljarrah, arafat.dweik\}@ku.ac.ae).

A. Al-Dweik is also with the Department of Electrical and Computer Engineering, London, Western University, ON, Canada. (E-mail: dweik@fulbrightmail.org).

K.-H Park and M.-S. Alouini are with King Abdullah University of Science and Technology (KAUST), Thuwal, Makkah Province, Kingdom of Saudi Arabia, (E-mail: \{ kihong.park, slim.alouini\}@kaust.edu.sa). 


\section{INTRODUCTION}

Generally speaking, there are three main types of detection schemes for digital signals, which are coherent detection, noncoherent detection, and partially coherent detection [1]. The detector design, required channel state information (CSI), computational complexity, and symbol error rate (SER) of each detection scheme depend on several factors such as the modulation scheme, modulation order, and channel model. Therefore, adopting particular modulation and detection schemes is mostly determined by the targeted application. For example, broadband communications require spectrally efficient modulation schemes to support high data rates, and the communicating nodes typically have sufficient resources to estimate the CSI, and hence, modulation schemes with high order and coherent detectors are utilized. For most wireless applications, quadrature amplitude modulation (QAM) is considered as the most attractive due to its power and spectral efficiency [2][4]. Nevertheless, $M$-ary amplitude shift keying (MASK) has recently attracted extensive attention because it is more suitable for certain applications such as wireless sensor networks (WSNs) [5], wireless energy transfer [6], radio frequency identification (RFID) [7], and optical wireless communications (OWC) [8]-[12].

Unlike typical wireless communications systems, OWC such as free space optics (FSO) and visible light communications (VLC) that use intensity modulation with direct detection (IM-DD) require the baseband signal to be real and positive, and hence, using QAM for OWC directly is infeasible. To overcome this limitation, QAM can be combined with orthogonal frequency division multiplexing (OFDM) to generate real and positive signals using various techniques [13]. Nevertheless, the spectral efficiency and SER of QAM-OFDM are generally equivalent to MASKOFDM [14]. Therefore, MASK renders itself as an efficient alternative to QAM for OWC [15] because it can be used with/without OFDM. Moreover, in IM-DD, the binary ASK (BASK) can be detected using a simple noncoherent detector that does not require prior knowledge of the instantaneous CSI. The noncoherent BASK detector has low complexity and is robust to hardware impairments such as the carrier frequency offset and phase noise, but it suffers from poor spectral efficiency, and accurate knowledge of the statistical CSI is necessary to compute the optimum threshold. Improving the spectral efficiency of noncoherent MASK modulation by increasing the modulation order $M$ is not feasible in fading channels due to its poor SER [1], which limits its utilization to additive white Gaussian noise (AWGN) channels. Practically speaking, the AWGN 
channel model is limited to few applications such as indoor OWC [13].

Although the channel in OWC may be considered non-fading in certain scenarios [16], [17], channel models that consider the fading induced by atmospheric turbulence can be more practical, and they are actually more flexible because they can be used to describe a wide range of fading scenarios. In the literature, several channel models have been adopted for OWC including the Gamma-Gamma, exponential and Rician [13], [18], [19]. Moreover, the random pointing error in OWC are typically modeled as Rician [20]-[22]. The Rician channel model is of particular interest because it is also widely adopted in wireless communications systems, such as massive multiple-input multiple-output (MIMO) systems [23]-[27], satellite/drone to ground channels [28], [29], ${ }^{\left[C_{1}\right]}$ RFID [30] and WSNs [31]. Therefore, the Rician channel model is adopted in this work.

To resolve the spectral efficiency and SER conflict of MASK, Al-Dweik and Iraqi [1] recently proposed a semi-coherent detection scheme, also denoted as amplitude coherent (AC) detection, that allows using MASK modulation with $M>2$ over dispersive channels while maintaining the main advantages of noncoherent detection such as low receiver complexity, and immunity to phase noise and frequency offsets. The AC detector requires only the knowledge of the channel gain, which can be obtained blindly and efficiently for single and multicarrier modulation schemes [32], [33]. The channel phase information is not required, which is the main factor that contributes to the complexity reduction of the detector. The optimum, suboptimum and a heuristic detectors are derived in Rayleigh fading channels, and the performance of the heuristic detector is evaluated with and without perfect knowledge of the channel gain in [1] and [32], respectively. The optimum amplitudes of the transmitted MASK symbols are then derived for multibranch detectors in [34]. However, the Rayleigh fading model is limited to wireless applications with no line-of-sight (LoS) signal component. Therefore, applying the AC detector and evaluating its performance in a more general channel model is indispensable.

Consequently, this paper considers applying the AC detection technique to communication systems in Rician fading channels with single and multiple receiving antennas. More specifically, the optimum AC detector is derived, and its SER is compared with the optimum coherent and noncoherent MASK detectors. Moreover, efficient expressions for the SER and asymptotic SER at high signal-to-noise ratios (SNRs) are derived for the heuristic detector [1]. The obtained analytical and simulation results demonstrate that the AC detector can offer reliable SER performance that 
is comparable to coherent detection in Rician fading channels.

The notations used throughout this paper are as follows. Bold upper and lower case letters and symbols such as $\mathbf{x}, \mathbf{X}, \phi$ and $\mathbf{\Phi}$ are used to denote vectors, blackboard fonts such as $\mathbb{S}$ are used to denote sets. Variables and numbers normalized by the total noise variance such as $1 / 2 \sigma_{n}^{2}$ and $\beta / 2 \sigma_{n}^{2}$ are denoted as $\overline{1}$ and $\bar{\beta}$, respectively. Trial values in a maximization or minimization problem are denoted using a tilde, for example, the trial values for $a$ are denoted as $\tilde{a}$.

The rest of the paper is organized as follows. Section II presents the model of $M$-ary ASK system. In section III, the different types of considered detectors are derived including coherent, noncoherent and AC detectors. Sections IV presents the analysis of the SER for the heuristic AC detector. Sections V and VI provide the numerical results and conclusion, respectively.

\section{System And Channel Models}

In unipolar MASK modulation, the baseband representation of the transmitted signal during the $\ell$ th signaling interval is given by

$$
d^{\{\ell\}}=s_{m}, m \in\{0,1, \ldots, M-1\},
$$

where $M$ is the modulation order, the transmitted symbols $s_{m} \in \mathbb{R}$, where $\mathbb{R}$ is the set of nonnegative integers. Without loss of generality, the symbols' amplitudes can be ordered such that $s_{m+1}>s_{m}$. Moreover, the amplitude spacing is assumed to be uniform such that $s_{m+1}-s_{m}=\delta$.

It should be noticed that $\frac{1}{M} \sum_{m=0}^{M-1} E_{m}=1, E_{m}=s_{m}^{2}$, when the average symbol energy is normalized to unity. Therefore, the transmitted symbol during the $\ell$ th transmission interval can be described by,

$$
d^{\{\ell\}}=m \times \delta, \quad m \in\{0,1, \ldots, M-1\},
$$

where $m$ is selected uniformly, and $\delta$ is defined as [1]

$$
\delta=\sqrt{\frac{6}{(2 M-1)(M-1)}} .
$$

The system under consideration assumes that the transmitter is equipped with a single transmit antenna, and the receiver is equipped with $N$ receiving antennas. The channels between the transmitting and receiving antennas are assumed to be flat, independent and identically distributed 
(i.i.d.) Rician fading channels. Therefore, the received signals in vector notation can be written as

$$
\mathbf{r}=\mathbf{h} s_{m}+\mathbf{n}, m \in\{0,1, \ldots, M-1\}
$$

where the channel fading vector $\mathbf{h} \in \mathbb{C}^{N \times 1}, h_{i} \sim \mathcal{C N}\left(m_{h}, 2 \sigma_{h}^{2}\right)$ represents the Rician fading, $s_{m}$ is the information symbol selected uniformly from the set $\mathbb{S}=\left\{s_{0}, s_{1}, \ldots, s_{M-1}\right\}$, and the AWGN vector $\mathbf{n} \in \mathbb{C}^{N \times 1}$ where $n_{i} \sim \mathcal{C N}\left(0,2 \sigma_{n}^{2}\right)$. The received signal in (4) can also be written as

$$
\mathbf{r}=[\boldsymbol{\alpha} \circ \boldsymbol{\Phi}] s_{m}+\mathbf{n}
$$

where $\boldsymbol{\alpha}=\left[\left|h_{1}\right|,\left|h_{2}\right|, \ldots,\left|h_{N}\right|\right], \boldsymbol{\Phi} \triangleq e^{j \boldsymbol{\theta}}$, and $\circ$ denotes the Hadamard product. ${ }^{\left[C_{2}\right]}$ It is worth noting that the channel gain is modeled as a complex random variable to capture the amplitude and phase variations caused by fading channels, and the additive noise is modeled as complex random variable because of the quadrature receiver that is typically used in such systems [37, pp. $66]$.

\section{A. Rician Channel Model}

In Rician fading, the received signal has a LoS component that affects the received signal envelope and phase. After dropping the channel index, the joint probability density function (PDF) of the channel envelope $\alpha \triangleq|h|$ and and phase $\theta \triangleq \arg \{h\}$ is given by

$$
f(\alpha, \theta)=\frac{\alpha}{2 \pi \sigma_{h}^{2}} \exp \left(-\frac{\alpha^{2}-2 \mu_{h} \alpha \cos \theta+\mu_{h}^{2}}{2 \sigma_{h}^{2}}\right),
$$

where $\mu_{h}=\left|m_{h}\right|$. The marginal PDF of $\alpha$ can be obtained by averaging the joint PDF $f(\alpha, \theta)$ over $\theta$. Thus

$$
\begin{aligned}
f(\alpha) & =\int_{-\pi}^{\pi} f(\alpha, \theta) d \theta \\
& =\frac{2(1+K)}{\Omega} \alpha \mathrm{e}^{-K} \mathrm{e}^{-\frac{(1+K)}{\Omega} \alpha^{2}} I_{0}\left(2 \alpha \sqrt{\frac{K(1+K)}{\Omega}}\right),
\end{aligned}
$$

where $\mathrm{E}\left[\alpha^{2}\right] \triangleq \Omega=\mu_{h}^{2}+2 \sigma_{h}^{2}$ and $K=\frac{\mu_{h}^{2}}{2 \sigma_{h}^{2}}$ is the Rician factor that determines the link quality, $K \in(0, \infty)$. For small values of $K$, the channel fading becomes severe, which indicates that the link quality is poor, while high quality links are typically achieved for $K \gtrsim 10$ [35]. The PDF of 
the phase $\theta$ can be obtained by averaging over the PDF of $\alpha$ [36], which gives

$$
f(\theta)=\frac{1}{2 \pi} \mathrm{e}^{-K}+\sqrt{\frac{K}{\pi}} \cos (\theta+\phi) \exp \left(-K \sin ^{2}(\theta+\phi)\right) Q(-\sqrt{2 K} \cos (\theta+\phi)),
$$

where $\phi=\tan ^{-1}\left(\frac{\mu_{h, Q}}{\mu_{h, I}}\right), \mu_{h, I} \triangleq \Re\left\{m_{h}\right\}, \mu_{h, Q}=\Im\left\{m_{h}\right\}$, and $Q(\cdot)$ is the $Q$-function.

\section{MASK DETECTOR DESIGN}

Usually, there is a trade-off between the receiver complexity and SER performance. The complexity may refer to the computational complexity, hardware complexity or the amount of information required at the receiver side. Adopting a certain detector design depends on the desired application. Other parameters such as the spectral efficiency can affect the complexity and SER. Because it is typically difficult to achieve such conflicting objectives simultaneously, it is crucial to have various options that may fit various applications. In this section, various optimum and suboptimum detectors are derived for MASK signals in Rician fading channels, and their complexity will be discussed.

\section{A. Coherent Detection}

Based on the signal model in (4), and noting that all $N$ received signals are mutually independent, the conditional PDF of $\mathbf{r}$ for a given fading vector $\mathbf{h}$, and a transmitted symbol $s_{m}$ is given by [37]

$$
f\left(\mathbf{r} \mid \mathbf{h}, s_{m}\right)=\prod_{i=1}^{N} f\left(r_{i} \mid h_{i}, s_{m}\right)
$$

As can be noted from (4), $f\left(r_{i} \mid h_{i}, s_{m}\right) \sim \mathcal{C N}\left(h_{i} s_{m}, 2 \sigma_{n}^{2}\right)$, and thus (10) can be written as

$$
f\left(\mathbf{r} \mid \mathbf{h}, s_{m}\right)=\frac{1}{\left(2 \pi \sigma_{n}^{2}\right)^{N}} \prod_{i=1}^{N} \exp \left(-\overline{1}\left|r_{i}-h_{i} s_{m}\right|^{2}\right) .
$$

The maximum likelihood (ML) detector based on (11) can be formulated as

$$
\hat{d}=\arg \max _{\tilde{s}_{m} \in \mathbb{S}} f\left(\mathbf{r} \mid \mathbf{h}, \tilde{s}_{m}\right)
$$

where $\tilde{s}_{m}$ are the trial values of $s_{m}$. By taking the $\log$ of the objective function, dropping the common terms and constants we obtain,

$$
\hat{d}=\arg \min _{\tilde{s}_{m} \in \mathbb{S}} \sum_{i=1}^{N}\left|r_{i}-h_{i} \tilde{s}_{m}\right|^{2} .
$$


As can be noted from (13), the computational complexity of the coherent detector is low, however, the fading parameters represented by $\mathbf{h}$ should be available at the receiver. Generally speaking, estimating $\mathbf{h}$ requires significant efforts, and inaccurate channel estimation deteriorates the system SER [38].

\section{B. Noncoherent Detection}

The noncoherent detector can be derived following the same approach of the coherent detector, except that the detector should not have any information about the instantaneous values of $\mathbf{h}$. Consequently, $\mathbf{h}$ should be treated as a random vector. In such cases, the ML detector can be formulated as

$$
\begin{aligned}
\hat{d} & =\arg \max _{\tilde{s}_{m} \in \mathbb{S}} f\left(\mathbf{r} \mid \tilde{s}_{m}\right) \\
& =\arg \max _{\tilde{s}_{m} \in \mathbb{S}} \prod_{i=1}^{N} f\left(r_{i} \mid \tilde{s}_{m}\right) .
\end{aligned}
$$

The conditional PDF $f\left(r_{i} \mid s_{m}\right)$ is a complex Gaussian random variable, and thus

$$
f\left(r_{i} \mid s_{m}\right)=\frac{1}{\pi \sigma_{\mathrm{r}}^{2}} \exp \left(-\frac{\left|r_{i}-\mu_{h} s_{m}\right|^{2}}{\sigma_{\mathrm{r}}^{2}}\right) .
$$

where $\sigma_{\mathrm{r}}^{2} \triangleq 2\left(\sigma_{h}^{2} s_{m}^{2}+\sigma_{n}^{2}\right)$. After some straightforward simplifications, the ML noncoherent detector reduces to

$$
\hat{d}=\arg \min _{\tilde{s}_{m} \in \mathbb{R}}\left\{-N \ln \left(\pi \tilde{\sigma}_{\mathrm{r}}^{2}\right)+\frac{1}{\tilde{\sigma}_{\mathrm{r}}^{2}} \sum_{i=1}^{N}\left|r_{i}-\mu_{h} \tilde{s}_{m}\right|^{2}\right\},
$$

where $\tilde{\sigma}_{\mathrm{r}}^{2}=\left.\sigma_{\mathrm{r}}^{2}\right|_{s_{m} \rightarrow \tilde{s}_{m}}$. As can be noted from (16), the noncoherent detector does not require the knowledge of $\mathbf{h}$, instead, it requires the statistical channel information, i.e., the values of $\mu_{h}, \sigma_{h}^{2}$ and $\sigma_{n}^{2}$. Estimating the statistical information of the channel is generally challenging because it requires large number of observations, and hence, large delay and high computational complexity. Therefore, similar to the coherent detector, the ML noncoherent detector has complexity limitations as well.

\section{Amplitude Coherent Detection}

The AC detector is designed as a compromise between the poor SER of the noncoherent and the high complexity of the coherent detector caused by the channel estimation process [1]. More 
specifically, the AC detector is designed assuming that the receiver has partial knowledge about the channel, namely, the fading gains vector $\alpha$, but no information is required for $\Phi$. Since phase estimation is typically more complex to achieve as compared to the channel envelope, the AC detector complexity is less than the coherent detection [32]. The following subsections present the derivation of the optimum and suboptimum AC detectors.

1) Optimum AC Detector: The optimum AC detector can be derived by applying the ML criterion and assuming the phase shift introduced by the channel is unknown. Therefore,

$$
\hat{d}=\arg \max _{\tilde{s}_{m} \in \mathbb{S}} f\left(\mathbf{r} \mid \boldsymbol{\alpha}, \tilde{s}_{m}\right) .
$$

Because $r_{i} \forall i$ are mutually independent, then the conditional joint PDF of $\mathbf{r}$ given that only the channel gain $\boldsymbol{\alpha}$ is known, can be derived as

$$
f\left(\mathbf{r} \mid \boldsymbol{\alpha}, s_{m}\right)=\prod_{i=1}^{N} \int_{-\pi}^{\pi} f\left(r_{i} \mid \alpha_{i}, \theta_{i}, s_{m}\right) f\left(\theta_{i}\right) d \theta_{i},
$$

where $f\left(\theta_{i}\right)$ is given in (9). The integration over the PDF of $\theta$ in (18) is performed because the $\mathrm{AC}$ detector is designed to retrieve the information symbols without the knowledge of the channel phase. By noting that the real and imaginary parts of $r_{i}$ are independent, and dropping the index $i$ for notational simplicity, then $f\left(r_{i} \mid \alpha_{i}, \theta_{i}, s_{m}\right)$ can be written as

$$
\begin{aligned}
f\left(r \mid \alpha, \theta, s_{m}\right) & =f\left(r_{\Re} \mid \alpha, \theta, s_{m}\right) f\left(r_{\Im} \mid \alpha, \theta, s_{m}\right) \\
& =\frac{\overline{1}}{\pi} \exp \left[-\overline{1}\left(|r|^{2}+\alpha^{2} s_{m}^{2}\right)\right] \exp \left[2 \bar{\alpha} s_{m}\left(r_{\Im} \sin (\theta)+r_{\Re} \cos (\theta)\right)\right] \\
& =\frac{\overline{1}}{\pi} \exp \left[-\overline{1}\left(|r|^{2}+\alpha^{2} s_{m}^{2}\right)\right] \exp \left[2 \bar{\alpha} s_{m}|r| \cos \left(\theta-\theta_{\mathrm{r}}\right)\right],
\end{aligned}
$$

where $\theta_{\mathrm{r}} \triangleq \tan ^{-1}\left(r_{\Im} / r_{\Re}\right)$. Then, $f\left(r \mid \alpha, s_{m}\right)$ can be evaluated by substituting (9) and (19) into (18). However, the PDF of $\theta$ given in (9) consists of the product of a trigonometric, exponential and a $Q$-function, and hence, evaluating the integral in (18) is intractable. Consequently, the Von Mises (Tikhonov or circular normal) distribution, which is considered as an accurate approximation for the PDF in (9) [39], is used,

$$
f(\theta) \approx \frac{1}{2 \pi I_{0}(\mathcal{K})} \exp (\mathcal{K} \cos (\theta-\phi)),
$$


where $\mathcal{K}=2 \sqrt{K(K+1)}$. Therefore, the integral $\int_{-\pi}^{\pi} f\left(r \mid \alpha, \theta, s_{m}\right) f(\theta) d \theta$ in (18) can be evaluated as

$$
f\left(r \mid \alpha, s_{m}\right) \approx G(r) \exp \left(-\overline{1} \alpha^{2} s_{m}^{2}\right) \mathcal{I}_{\theta}
$$

where $G(r)$

$$
G(r)=\frac{\overline{1}}{\pi I_{0}(\mathcal{K})} \exp \left(-\overline{1}|r|^{2}\right)
$$

and

$$
\mathcal{I}_{\theta}=\frac{1}{2 \pi} \int_{-\pi}^{\pi} \exp (\mathcal{K} \cos (\theta-\phi)) \exp \left(2 \bar{\alpha} s_{m}|r| \cos \left(\theta-\theta_{\mathrm{r}}\right)\right) d \theta
$$

The factor $G(r)$ can be considered constant with respect to the maximization process in (17), thus, it is more convenient to separate it from the other terms. Moreover, for the special case where $s_{m}=0$, the PDF $f\left(r \mid \alpha, \theta, s_{0}\right)$ is independent of $\alpha$ and $\theta$. Thus,

$$
\begin{aligned}
f\left(r \mid \alpha, \theta, s_{0}\right) & =\frac{\overline{1}}{\pi} \exp \left[-\overline{1}|r|^{2}\right] \\
& =G(r) I_{0}(\mathcal{K}) .
\end{aligned}
$$

For $s_{m} \neq 0$, evaluating the integral $\mathcal{I}_{\theta}$ is actually intractable due to the existence of $\phi$ and $\theta_{\mathrm{r}}$. Consequently, $\mathcal{I}_{\theta}$ can be approximated using the Gauss-Chebyshev quadrature integration rule as shown in Appendix I, which is given by

$$
\begin{aligned}
\mathcal{I}_{\theta}=\frac{1}{L} \sum_{l=1}^{L} \exp \left[\left(\mathcal{K} \cos (\phi)+2 \bar{\alpha} s_{m}|r|\right.\right. & \left.\cos \left(\theta_{\mathrm{r}}\right)\right) \cos \left(\varphi+\pi \frac{2 l-1}{2 L}\right) \\
& \left.+\left(\mathcal{K} \sin (\phi)+2 \bar{\alpha} s_{m}|r| \sin \left(\theta_{\mathrm{r}}\right)\right) \sin \left(\varphi+\pi \frac{2 l-1}{2 L}\right)\right],
\end{aligned}
$$

where $L$ is the quadrature order, and

$$
\varphi=\tan ^{-1}\left(\frac{\mathcal{K} \sin (\phi)+2 \bar{\alpha} s_{m} \sin \left(\theta_{\mathrm{r}}\right)|r|}{\mathcal{K} \cos (\phi)+2 \bar{\alpha} s_{m} \cos \left(\theta_{\mathrm{r}}\right)|r|}\right) .
$$

Therefore, after applying the $\ln (\cdot)$ function and dropping the common and constant terms, the optimum AC detector reduces to

$$
\hat{d}=\arg \min _{\tilde{s}_{m} \in \mathbb{S}} \sum_{i=1}^{N} \overline{1} \alpha_{i}^{2} \tilde{s}_{m}^{2}-\ln \tilde{\mathcal{I}}_{\theta_{i}},
$$

where $\tilde{\mathcal{I}}_{\theta_{i}}=\left.\mathcal{I}_{\theta_{i}}\right|_{s_{m} \rightarrow \tilde{s}_{m}}$, and the received signal phase can be computed directly from the received signal, $\theta_{\mathrm{r}} \triangleq \tan ^{-1}\left(r_{\Im} / r_{\Re}\right)$. As can be noted from (27), the optimum AC detector has very high computational complexity induced by $\mathcal{I}_{\theta_{i}}$, which makes it prohibitively expensive to implement. 
Moreover, the detector requires the knowledge of the noise variance $\sigma_{n}^{2}$ and the Rician fading parameter $K$. By noting that the detector in (27) is derived using an approximated $f(\theta)$ and $\mathcal{I}_{\theta}$, then it is denoted as near-optimum detector. It is also worth noting that the accuracy of $\mathcal{I}_{\theta}$ is mostly determined by the SNR because the second exponent in (23) may experience enormously high values at high SNRs, which might affect the integral accuracy.

2) Suboptimum AC Detector: As can be noted from (64) in Appendix I, $B$ and $D$ dominates $g(\theta)$ at high SNRs, i.e., $B \gg A \cos (\phi)$ and $D \gg C$. Thus, substituting $A=C=0$ in (64) yields

$$
\begin{aligned}
\mathcal{I}_{\theta} & \approx \frac{1}{2 \pi} \int_{-\pi}^{\pi} \exp \left(B \alpha s_{m}|r| \cos (\theta)+D \alpha s_{m}|r| \sin (\theta)\right) d \theta \\
& =\frac{1}{2 \pi} \int_{-\pi}^{\pi} \exp \left(2 \bar{\alpha} s_{m}|r|\left[\cos \left(\theta_{\mathrm{r}}\right) \cos (\theta)+\sin \left(\theta_{\mathrm{r}}\right) \sin (\theta)\right]\right) d \theta \\
& =\frac{1}{2 \pi} \int_{-\pi}^{\pi} \exp \left(2 \bar{\alpha} s_{m}|r| \cos \left(\theta-\theta_{\mathrm{r}}\right)\right) d \theta .
\end{aligned}
$$

Using the same approach that is used to derive (25), and the fact that the integrand in (28) is periodic with respect to $\theta$, then $\theta_{\mathrm{r}}$ can be dropped without affecting the integral. Consequently,

$$
\begin{aligned}
\mathcal{I}_{\theta} & \approx \frac{1}{\pi} \int_{0}^{\pi} \exp \left(2 \bar{\alpha} s_{m}|r| \cos (\theta)\right) d \theta \\
& =I_{0}\left(2 \bar{\alpha} s_{m}|r|\right) .
\end{aligned}
$$

Therefore, the AC detector can be expressed as

$$
\hat{d}=\arg \min _{\tilde{s}_{m} \in \mathbb{S}} \sum_{i=1}^{N} \overline{1} \alpha_{i}^{2} \tilde{s}_{m}^{2}-\ln \left[I_{0}\left(2 \bar{\alpha}_{i} \tilde{s}_{m}\left|r_{i}\right|\right)\right] .
$$

It is worth noting that the suboptimum AC detector described in (30) is similar to the optimum

AC detector derived in [1] for Rayleigh fading channels. Although this detector does not require knowledge of the statistical channel information, it requires the knowledge of the AWGN variance $\sigma_{n}^{2}$, and computing the Bessel function, which incurs high complexity.

Another suboptimal detector can be obtained by noting that $A \ll B$ and $C \ll D$ (64), therefore $\mathcal{I}_{\theta}$ can be approximated as

$$
\begin{aligned}
\mathcal{I}_{\theta} & \approx \frac{1}{L} \sum_{l=1}^{L} \exp \left[2 \bar{\alpha} s_{m}|r|\left(\cos \left(\theta_{\mathrm{r}}\right) \cos \left(\varphi+\pi \frac{2 l-1}{2 L}\right)+\sin \left(\theta_{\mathrm{r}}\right) \sin \left(\varphi+\pi \frac{2 l-1}{2 L}\right)\right)\right] \\
& =\frac{1}{L} \sum_{l=1}^{L} \exp \left[2 \bar{\alpha} s_{m}|r| \cos \left(\pi \frac{2 l-1}{2 L}\right)\right]
\end{aligned}
$$


Interestingly, this approach does not contain the Bessel function. Moreover, extensive simulation results demonstrated the SER for both approaches is equivalent, and hence, only the second approach will be considered in Section V.

3) Heuristic AC Detector: Although the two suboptimum AC detectors derived above are less complex than the optimum AC detector, evaluating the Bessel and exponential functions is necessary to calculate the decision metric. Therefore, the heuristic detector presented in [1] is considered to reduce the complexity even further. The heuristic detector is given by

$$
\hat{d}=\arg \min _{\tilde{s}_{m} \in \mathbb{S}}\left[\zeta-\tilde{s}_{m}^{2}\right]^{2},
$$

where $\zeta$ is the combined signal from the $N$ antennas, which is given by

$$
\zeta=\frac{|r|_{\Sigma}^{2}}{\sum_{i=1}^{N} \alpha_{i}^{2}},
$$

where $|r|_{\Sigma}^{2}=\sum_{i=1}^{N}\left|r_{i}\right|^{2}$. For SER analysis, it is more convenient to express (31) as

$$
\hat{d}=\left\{\begin{array}{cc}
s_{0}, & 0<\zeta<\eta_{0,1} \\
s_{1}, & \eta_{0,1}<\zeta<\eta_{1,2} \\
\vdots & \vdots \\
s_{M-1}, & \eta_{M-2, M-1}<\zeta<\infty
\end{array}\right.
$$

where $\eta_{i, j}$ 's are the detection thresholds given by

$$
\eta_{i, j}=\frac{1}{2}\left(s_{i}^{2}+s_{j}^{2}\right) .
$$

Based on the derived detectors, we summarize the various MASK detection schemes in Rician fading channels based on the required CSI. Table I shows the objective function (OF) of the detector using coherent (CO), noncoherent (NC) and AC detection. For the AC detection, the table presents the optimum (OP), suboptimum (SO) and heuristic $(\mathrm{H})$, while only the optimum is presented for the coherent and noncoherent detectors. As can be noted from the table, NC-OP is the only detector that does not require prior knowledge of the any instantaneous CSI parameter, instead, it requires the knowledge of statistical information of the channel, i.e., $\sigma_{h}$ and $\mu_{h}$, of the fading channel and $\sigma_{n}$ of the AWGN. Although the complexity of the detector itself is low, the estimation of the channel statistics is not, because it requires performing several operations over a large number of received samples. The CO-OP detector has the lowest complexity, does 
TABLE I

DIFFERENT MASK DETECTION SCHEMES AND CSI REQUIREMENTS.

\begin{tabular}{l|l|c|c|c}
\hline \hline & \multicolumn{2}{|c|}{ Rician } & \multicolumn{1}{c}{ Rayleigh [1] } \\
\hline \hline Type & \multicolumn{1}{|c|}{ Detector OF } & CSI & Detector OF & CSI \\
\hline \hline CO-OP & $\sum_{i=1}^{N}\left|r_{i}-h_{i} \tilde{s}_{m}\right|^{2}(13)$ & $\alpha, \theta$ & $\sum_{i=1}^{N}\left|r_{i}-h_{i} \tilde{s}_{m}\right|^{2}$ & $\alpha, \theta$ \\
\hline \hline $\mathrm{NC}-\mathrm{OP}$ & $-N \ln \left(\pi \tilde{\sigma}_{\mathrm{r}}^{2}\right)+\frac{1}{\tilde{\sigma}_{\mathrm{r}}^{2}} \sum_{i=1}^{N}\left|r_{i}-\mu_{h} \tilde{s}_{m}\right|^{2}(16)$ & $\sigma_{h}, \mu_{h}, \sigma_{n}$ & $-N \ln \left(\pi \tilde{\sigma}_{\mathrm{r}}^{2}\right)+\frac{1}{\tilde{\sigma}_{\mathrm{r}}^{2}} \sum_{i=1}^{N}\left|r_{i}\right|^{2}$ & $\sigma_{h}, \sigma_{n}$ \\
\hline \hline $\mathrm{AC}-\mathrm{OP}$ & $\sum_{i=1}^{N} \bar{\alpha}_{i}^{2} \tilde{s}_{m}^{2}-\ln \tilde{\mathcal{I}}_{\theta_{i}}(27)$ & $\alpha, \sigma_{h}, \mu_{h}, \sigma_{n}$ & $\sum_{i=1}^{N} \bar{\alpha}_{i}^{2} \tilde{s}_{m}^{2}-\ln \left[I_{0}\left(2 \bar{\alpha}_{i} \tilde{s}_{m}\left|r_{i}\right|\right)\right]$ & $\alpha$ \\
\hline $\mathrm{AC}-\mathrm{SO}$ & $\sum_{i=1}^{N} \bar{\alpha}_{i}^{2} \tilde{s}_{m}^{2}-\ln \left[I_{0}\left(2 \bar{\alpha}_{i} \tilde{s}_{m}\left|r_{i}\right|\right)\right](30)$ & $\alpha, \sigma_{n}$ & $\sum_{i=1}^{N}\left[\left|r_{i}\right|-\alpha_{i} \tilde{s}_{m}\right]^{2}$ & $\alpha$ \\
\hline $\mathrm{AC}-\mathrm{H}$ & {$\left[\frac{|r|_{\Sigma}^{2}}{\sum_{i=1}^{N} \alpha_{i}^{2}}-\tilde{s}_{m}^{2}\right]^{2}(31)$} & $\alpha$ & {$\left[\frac{|r|_{\Sigma}^{2}}{\sum_{i=1}^{N} \alpha_{i}^{2}}-\tilde{s}_{m}^{2}\right]^{2}$} & $\alpha$ \\
\hline
\end{tabular}

not require channel statistical information, but it requires accurate estimates of the instantaneous channel phase and gain. The AC-OP has generally the highest complexity due to $\tilde{\mathcal{I}}_{\theta_{i}}$, moreover, it requires instantaneous and statistical channel information. The AC-SO has low complexity, but it requires instantaneous and statistical channel information. Finally, the $\mathrm{AC}-\mathrm{H}$ is the only detector that requires the knowledge of a single parameter, which is the instantaneous channel gain $\alpha$. Furthermore, by comparing the same types of detectors in Rayleigh fading channels, it can be noted that the detectors' complexity in Rayleigh fading channels is less than the Rician, and the CSI requirements are less in all cases except for the $\mathrm{CO}-\mathrm{OP}$ and the $\mathrm{AC}-\mathrm{H}$. In fact, the CO-OP and AC-H detectors' design is independent of the channel model.

\section{Phase Coherent Detection}

The phase coherent (PC) detector is designed assuming that the only the channel phase vector $\Phi$ is available at the receiver. Therefore, the PC detector can be formulated as

$$
\begin{aligned}
\hat{d} & =\arg \max _{\tilde{s}_{m} \in \mathbb{S}} \ln f\left(\mathbf{r} \mid \mathbf{\Phi}, \tilde{s}_{m}\right) \\
& =\sum_{i=1}^{N} \ln \int_{0}^{\infty} f\left(r_{i} \mid \alpha_{i}, \theta_{i}, s_{m}\right) f\left(\alpha_{i}\right) d \alpha_{i}
\end{aligned}
$$

where $f\left(\alpha_{i}\right)$ and $f\left(r_{i} \mid \alpha_{i}, \theta_{i}, s_{m}\right)$ are given in (8) and (19), respectively. However, similar to the AC optimum detector, the integral in (35) does not have a closed-form solution. Therefore, we use equal gain combining as a heuristic detector for the MASK PC detection. Thus, the combined 
received signal can be written as

$$
r_{\Sigma}=\frac{1}{N} \sum_{i=1}^{N} r_{i} \mathrm{e}^{-j \theta_{i}} .
$$

Because the data part in (36) after co-phasing is real, the real part of $r_{\Sigma}$ is extracted and then applied to a conventional coherent MASK detector, except that the thresholds are computed to minimize $P_{\mathrm{e}}$ using Mote Carlo simulation.

\section{SER AnAlysis of The Heuristic AC Detector}

Due to the non-symmetry of the SER $\left(P_{\mathrm{e}}\right)$ with respect to the transmitted symbols, the SER for equiprobable transmitted symbols ${ }^{\left[C_{3}\right]}$ for the detector described in (33) can be written as $P_{\mathrm{e}}=\frac{1}{M} \sum_{m=0}^{M-1} P_{\mathrm{e}} \mid s_{m}=\frac{1}{M} \sum_{m=0}^{M-1}\left(1-P_{C} \mid s_{m}\right)$, where $P_{C} \mid s_{m}$ and $P_{\mathrm{e}} \mid s_{m}$ are the conditional probability of correct and erroneous decisions given that symbol $s_{m}$ is transmitted. Therefore,

$$
P_{C} \mid s_{m}= \begin{cases}\operatorname{Pr}\left(\zeta<\eta_{0,1}\right), & m=0 \\ \operatorname{Pr}\left(\eta_{m-1, m}<\zeta<\eta_{m, m+1}\right), & m=1,2, \ldots, M-2 . \\ \operatorname{Pr}\left(\zeta>\eta_{M-2, M-1}\right), & m=M-1\end{cases}
$$

Therefore,

$$
\begin{array}{r}
P_{C}=\frac{1}{M}\left[\int_{0}^{\eta_{0,1}} f\left(\zeta \mid E_{0}\right) d \zeta+\sum_{m=1}^{M-2} \int_{\eta_{m, m-1}}^{\eta_{m, m+1}} f\left(\zeta \mid E_{m}\right) d \zeta+\int_{\eta_{M-2, M-1}}^{\infty} f\left(\zeta \mid E_{M-1}\right) d \zeta\right] \\
=\frac{1}{M}\left[F_{\zeta}\left(\eta_{0,1} \mid E_{0}\right)+\sum_{m=1}^{M-2}\left[F_{\zeta}\left(\eta_{m, m+1} \mid E_{m}\right)-F_{\zeta}\left(\eta_{m, m-1} \mid E_{m}\right)\right]\right] \\
+\frac{1}{M}\left(1-F_{\zeta}\left(\eta_{M-2, M-1} \mid E_{m}\right)\right)
\end{array}
$$

where $F_{\zeta}\left(\zeta \mid E_{m}\right)$ is the conditional cumulative distribution function (CDF). Therefore, the evaluation of $P_{\mathrm{e}}$ requires the evaluation of $F_{\zeta}\left(\zeta \mid E_{m}\right)$.

In this section, we derive the conditional $\mathrm{CDF} F_{\zeta}\left(\zeta \mid \beta, E_{m}\right), \beta=\sum_{i=1}^{N} \alpha_{i}^{2}$ from the conditional $\operatorname{PDF} f\left(\zeta \mid \beta, E_{m}\right)$, and then we derive the unconditional $\operatorname{CDF} F_{\zeta}\left(\zeta \mid E_{m}\right)$. By noting that $\zeta=\frac{|r|_{\Sigma}^{2}}{\beta}$, then the conditional PDF $f\left(\zeta \mid \beta, E_{m}\right)$ follows a noncentral Chi-squared with $2 N$ degrees of freedom, noncentrality parameter $E_{m}$, and the variance of Gaussian components is $\frac{\sigma_{n}^{2}}{\beta}$, i.e., $f\left(\zeta \mid \beta, E_{m}\right) \sim \chi^{2}\left(2 N, s_{m}^{2}, \frac{\sigma_{n}^{2}}{\beta}\right)=\chi^{2}\left(2 N, E_{m}, \frac{\sigma_{n}^{2}}{\beta}\right)$. To derive $f\left(\zeta \mid \beta, E_{m}\right)$ of the Heuristic 
detector (31), the decision variable can be written as

$$
\begin{aligned}
\zeta & =\frac{|r|_{\Sigma}^{2}}{\sum_{i=1}^{N} \alpha_{i}^{2}} \\
& =\frac{1}{\sum_{i=1}^{N} \alpha_{i}^{2}} \sum_{i=1}^{N}\left|r_{i}\right|^{2} \\
& =\frac{1}{\sum_{i=1}^{N} \alpha_{i}^{2}} \underbrace{\sum_{i=1}^{N}\left|r_{i, \Re}+j r_{i, \Im}\right|^{2}}_{r_{T}},
\end{aligned}
$$

where $r_{i, \Re}=\alpha_{i} s_{m} \cos \left(\theta_{i}\right)+n_{i, \Re}$ and $r_{i, \Im}=\alpha_{i} s_{m} \sin \left(\theta_{i}\right)+n_{i, \Im}$. For a given $\alpha_{i}, s_{m}$ and $\theta_{i}$, the variables $r_{i, \Re}$ and $r_{i, \Im}$ are mutually independent $\forall i$, and the PDF for each of which is conditionally Gaussian, i.e., $f\left(r_{i, \Re} \mid \alpha_{i}, s_{m}, \cos \left(\theta_{i}\right)\right) \sim \mathcal{N}\left(\alpha_{i} s_{m} \cos \left(\theta_{i}\right), \sigma_{n}^{2}\right)$ and $f\left(r_{i, \Im} \mid \alpha_{i}, s_{m}, \sin \left(\theta_{i}\right)\right) \sim$ $\mathcal{N}\left(\alpha_{i} s_{m} \sin \left(\theta_{i}\right), \sigma_{n}^{2}\right)$. Therefore, $f\left(\left|r_{i}\right|^{2} \mid \alpha_{i}, s_{m}, \theta_{i}\right)$ is conditionally noncentral Chi-squared with two degrees of freedom and noncentrality factor $\lambda_{i}$, i.e., $f\left(\left|r_{i}\right|^{2} \mid \alpha_{i}, s_{m}, \theta_{i}\right) \sim \chi^{2}\left(2, \lambda_{i}, \sigma_{n}^{2}\right)$, where

$$
\begin{aligned}
\lambda_{i} & =\left[\alpha_{i} s_{m} \cos \left(\theta_{i}\right)\right]^{2}+\left[\alpha_{i} s_{m} \cos \left(\theta_{i}\right)\right]^{2} \\
& =\alpha_{i}^{2} s_{m}^{2}
\end{aligned}
$$

Therefore, $f\left(\left|r_{i}\right|^{2} \mid \alpha_{i}, s_{m}, \theta_{i}\right)=f\left(\left|r_{i}\right|^{2} \mid \alpha_{i}, s_{m}\right) \sim \chi^{2}\left(2, \lambda_{i}, \sigma_{n}^{2}\right)$. Therefore, $f(\zeta \mid \beta)$ can be written as

$$
f\left(\zeta \mid \beta, E_{m}\right)=\bar{\beta}\left(\frac{\zeta}{E_{m}}\right)^{\frac{N-1}{2}} \exp \left(-\bar{\beta}\left[\zeta+E_{m}\right]\right) I_{N-1}\left(2 \bar{\beta} \sqrt{E_{m} \zeta}\right), m>0 .
$$

For $m=0, r_{i, \Re}=n_{i, \Re} \sim \mathcal{N}\left(0, \sigma_{n}^{2}\right)$ and $r_{i, \Im}=n_{i, \Im} \sim \mathcal{N}\left(0, \sigma_{n}^{2}\right)$, thus, $f\left(\left|r_{i}\right|^{2} \mid \alpha_{i}, s_{m}, \theta_{i}\right)=$ $f\left(\left|r_{i}\right|^{2}\right)$ which has exponential PDF with parameter $2 \sigma_{n}^{2}$. Consequently, the PDF of the sum of $N$ i.i.d. exponential random variables has Erlang distribution, i.e.,

$$
f\left(\zeta \mid \beta, E_{0}\right)=\frac{\bar{\beta}^{N}}{(N-1) !} \zeta^{N-1} \exp (-\zeta \bar{\beta}) .
$$

Thus, $F_{\zeta}\left(\zeta \mid \beta, E_{m}\right)[40]$ can be defined as

$$
F_{\zeta}\left(\zeta \mid \beta, E_{m}\right)=1-Q_{N}\left(\sqrt{2 \bar{\beta} E_{m}}, \sqrt{\bar{\beta} \zeta}\right) .
$$

where $Q_{N}(\cdot, \cdot)$ is the generalized Marcum $Q$ function. The CDF of $\zeta$ given $E_{m}$ can be calculated by averaging the conditional $\operatorname{CDF} F\left(\zeta \mid \beta, E_{m}\right)$ over the distribution of $\beta$ which is given by

$$
F_{\zeta}\left(\zeta \mid E_{m}\right)=\int_{0}^{\infty} F_{\zeta}\left(\zeta \mid \beta, E_{m}\right) f(\beta) d \beta
$$


The PDF $f(\beta)$ can be derived by noting that $h_{i} \sim \mathcal{C N}\left(m_{h}, 2 \sigma_{h}^{2}\right)$, thus $f(\beta)$ is noncentral Chisquared,

$$
f(\beta)=\frac{\exp \left(-\frac{\lambda}{2}\right)}{2 \sigma_{h}^{2}} \exp \left(-\frac{\beta}{2 \sigma_{h}^{2}}\right)\left(\frac{\beta}{\lambda \sigma_{h}^{2}}\right)^{0.5(N-1)} I_{N-1}\left(\sqrt{\frac{\lambda}{\sigma_{h}^{2}} \beta}\right),
$$

where $\lambda=\sum_{i=1}^{N} \mu_{h_{I, i}}^{2}+\mu_{h_{Q, i}}^{2}$, and because the $N$ channels are assumed to be i.i.d., then

$$
\begin{aligned}
\lambda & =N\left(\frac{\mu_{h}^{2} \cos ^{2} \phi}{\sigma_{h}^{2}}+\frac{\mu_{h}^{2} \sin ^{2} \phi}{\sigma_{h}^{2}}\right) \\
& =2 K N .
\end{aligned}
$$

It is worth noting that the conditional PDF $f\left(\zeta \mid E_{m}\right)$ for $m>0$ and $f\left(\zeta \mid E_{0}\right)$ are given in [41, Eq. 46] and [41, Eq. 54], respectively.

\section{A. The $C D F F_{\zeta}\left(\zeta \mid E_{m}\right)$ for $\zeta<E_{m}$}

The series representation of the generalized Marcum Q-function is given in [42] as

$$
Q_{v}(a, b)=1-\sum_{n=0}^{\infty}(-1)^{n} \exp \left(-\frac{a^{2}}{2}\right) \frac{L_{n}^{(v-1)}\left(\frac{a^{2}}{2}\right)}{\Gamma(v+n+1)}\left(\frac{b^{2}}{2}\right)^{n+v},\{a, v\}>0 \text { and } b \geq 0
$$

where $L_{n}^{(m)}(\beta)=\sum_{k=0}^{n} \frac{\Gamma(n+m+1)}{\Gamma(k+m+1) \Gamma(n-k+1)} \frac{(-\beta)^{k}}{k !}$ is the generalized Laguerre polynomial of degree $n$ and order $m$, and $\Gamma(\cdot)$ is the gamma function. Using this formula, the conditional CDF $F\left(\zeta \mid \beta, E_{m}\right)$ can be rewritten as

$$
F_{\zeta}\left(\zeta \mid \beta, E_{m}\right)=\sum_{n=0}^{\infty} \sum_{k=0}^{n}\left(\begin{array}{l}
N+n-1 \\
N+k-1
\end{array}\right) \frac{(-1)^{n+k}}{(N+n) ! k !}(\bar{\zeta})^{N+n} \bar{E}_{m}^{k} \beta^{N+n+k} \exp \left(-\bar{E}_{m} \beta\right) .
$$

Then, the $\mathrm{CDF} F_{\zeta}\left(\zeta \mid E_{m}\right)$ can be computed as

$$
F_{\zeta}\left(\zeta \mid E_{m}\right)=\sum_{n=0}^{\infty} \sum_{k=0}^{n}\left(\begin{array}{c}
N+n-1 \\
N+k-1
\end{array}\right) \frac{(-1)^{n+k}}{(N+n) ! k !}(\bar{\zeta})^{N+n}\left(\bar{E}_{m}\right)^{k} \underbrace{\int_{0}^{\infty} \beta^{N+n+k} e^{-\bar{E}_{m} \beta} f(\beta) d \beta}_{\dot{A}} .
$$

The integration $A ́$ can be solved using [43, Eq. 2.15.5]

$$
\begin{aligned}
\dot{A} & =\frac{\mathrm{e}^{-\frac{\lambda}{2}}}{\sigma_{h}^{2}}\left(\frac{1}{\lambda \sigma_{h}^{2}}\right)^{\frac{N-1}{2}} \int_{0}^{\infty} \beta^{2(N+n+k)+N} \exp \left(-\left(\bar{E}_{m}+\frac{1}{2 \sigma_{h}^{2}}\right) \beta^{2}\right) I_{N-1}\left(\sqrt{\frac{\lambda}{\sigma_{h}^{2}}} \beta\right) d \beta \\
& =\frac{\Gamma(n+k+2 N)}{\left(2 \sigma_{h}^{2}\right)^{N} \Gamma(N) \mathrm{e}^{\frac{\lambda}{2}}}\left(\bar{E}_{m}+\frac{1}{2 \sigma_{h}^{2}}\right)^{-(n+k+2 N)}{ }_{1} F_{1}\left(n+k+2 N ; N ; \frac{0.5 \lambda}{1+2 \sigma_{h}^{2} \bar{E}_{m}}\right), \quad
\end{aligned}
$$

where ${ }_{1} F_{1}(a ; b ; z)$ is the confluent hypergeometric function of the first kind.

Consequently, by substituting $A$ into (49) and applying some manipulations, the CDF can be 
expressed as

$$
\begin{aligned}
F_{\zeta}\left(\zeta \mid E_{m}\right)=\sum_{n=0}^{\infty} \sum_{k=0}^{n}\left(\begin{array}{l}
N+n-1 \\
N+k-1
\end{array}\right) & \frac{(-1)^{n+k}(n+k+2 N-1) !}{(N-1) !(N+n) ! k !}\left(\frac{\zeta}{E_{m}}\right)^{n+N}\left(K_{m} \mathrm{e}^{-K}\right)^{N} \\
& \times \frac{\bar{\Omega}^{n+k+N}}{\left(\bar{\Omega}+K_{m}\right)^{n+k+2 N}} 1 F_{1}\left(n+k+2 N ; N ; \frac{N K K_{m}}{\bar{\Omega}+K_{m}}\right),
\end{aligned}
$$

where $K_{m}=\frac{1+K}{E_{m}}$. Note that $\left(\frac{\zeta}{E_{m}}\right)^{n}$ in the CDF expression is converged only with $\frac{\zeta}{E_{m}}<1$ when $n$ goes to infinity. Therefore, it will be used to calculate the error probability for $\operatorname{Pr}\left(\zeta \leq \frac{E_{m}+E_{m-1}}{2}\right)$.

For high SNR regime, when $\bar{\Omega} \rightarrow \infty$, (51) can be expressed as

$$
\lim _{\bar{\Omega} \rightarrow \infty}\left(\frac{\bar{\Omega}}{\bar{\Omega}+K_{m}}\right)^{n+k+N}{ }_{1} F_{1}\left(n+k+2 N ; N ; \frac{N K K_{m}}{\bar{\Omega}+K_{m}}\right)=1 .
$$

Then, (51) can be simplified as

$$
\begin{aligned}
F_{\zeta}^{\infty}\left(\zeta \mid E_{m}\right) & =\left(\frac{K_{m} e^{-K}}{\bar{\Omega}+K_{m}}\right)^{N} \sum_{n=0}^{\infty}\left(\frac{\zeta}{E_{m}}\right)^{n+N} \sum_{k=0}^{n}\left(\begin{array}{c}
N+n-1 \\
N+k-1
\end{array}\right) \frac{(-1)^{n+k}(n+k+2 N-1) !}{(N-1) !(N+n) ! k !} \\
& =\left(\frac{K_{m} e^{-K}}{\bar{\Omega}+K_{m}}\right)^{N} \sum_{n=0}^{\infty}\left(\frac{\zeta}{E_{m}}\right)^{n+N} \frac{(2 N+n-1) !}{n ! N !(N-1) !} \\
& =\left(\begin{array}{c}
2 N-1 \\
N
\end{array}\right)\left(\frac{K_{m} e^{-K}}{\bar{\Omega}+K_{m}} \frac{\frac{\zeta}{E_{m}}}{\left(\frac{\zeta}{E_{m}}-1\right)^{2}}\right)^{N},\left(\zeta<E_{m}\right)
\end{aligned}
$$

where (52a) comes from

$$
\sum_{k=0}^{n}(-1)^{n+k}\left(\begin{array}{l}
N+n-1 \\
N+k-1
\end{array}\right) \frac{(n+k+2 N-1) !}{k !}=\frac{(n+2 N-1) !(n+N) !}{n ! N !}
$$

and (52b) comes from

$$
\sum_{n=0}^{\infty} a^{n} \frac{(n+2 N-1) !}{n !}=(a-1)^{-2 N}(2 N-1) !, a<1 .
$$

B. The CDF $F_{\zeta}\left(\zeta \mid E_{m}\right)$ for $\zeta>E_{m}$

To evaluate the required CDF, the following relation between $Q_{m}(a, b)$ and $Q_{m}(b, a)$ is applied.

$$
Q_{m}(a, b)+Q_{m}(b, a)=1+e^{-\frac{a^{2}+b^{2}}{2}} \sum_{k=1-m}^{m-1}\left(\frac{a}{b}\right)^{k} I_{k}(a b) .
$$

Using this transformation, the $\operatorname{CDF} F_{\zeta}\left(\zeta \mid \beta, E_{m}\right)$ for $\zeta>E_{m}$ can be now rewritten as

$$
F_{\zeta}\left(\zeta \mid \beta, E_{m}\right)=Q_{N}\left(\sqrt{2 \bar{\beta} \zeta}, \sqrt{2 \bar{\beta} E_{m}}\right)-\exp \left(-\left[\zeta+E_{m}\right] \bar{\beta}\right) \sum_{k=1-N}^{N-1}\left(\frac{E_{m}}{\zeta}\right)^{\frac{k}{2}} I_{k}\left(2 \sqrt{E_{m} \zeta} \bar{\beta}\right) .
$$


The CDF of $F_{\zeta}\left(\zeta \mid E_{m}\right)$ can be calculated as

$$
\begin{aligned}
F_{\zeta}\left(\zeta \mid E_{m}\right)= & \underbrace{\int_{0}^{\infty} Q_{m}\left(\sqrt{2 \bar{\beta} \zeta}, \sqrt{2 \bar{\beta} E_{m}}\right) f(\beta) d \beta}_{\dot{B}} \\
& -\underbrace{\sum_{k=1-N}^{N-1}\left(\frac{E_{m}}{\zeta}\right)^{\frac{k}{2}} \int_{0}^{\infty} \exp \left(-\left[\zeta+E_{m}\right] \bar{\beta}\right) I_{k}\left(2 \sqrt{E_{m} \zeta} \bar{\beta}\right) f(\beta) d \beta}_{\dot{C}},
\end{aligned}
$$

where integrals $\dot{B}$ and $C^{\prime}$ can be evaluated as shown below in (56) and (58), respectively. Integration in $B$ can be calculated similarly as (51) which is given by

$$
\begin{aligned}
\dot{B}=1-\sum_{n=0}^{\infty} \sum_{k=0}^{n}\left(\begin{array}{l}
N+n-1 \\
N+k-1
\end{array}\right) \frac{(-1)^{n+k}(n+k+2 N-1) !}{(N-1) !(N+n) ! k !}\left(\frac{E_{m}}{\zeta}\right)^{n+N}\left(K_{\zeta} \mathrm{e}^{-K}\right)^{N} \\
\quad \times \frac{\bar{\Omega}^{n+k+N}}{\left(\bar{\Omega}+K_{\zeta}\right)^{n+k+2 N}{ }_{1} F_{1}}\left(n+k+2 N ; N ; \frac{N K K_{\zeta}}{\bar{\Omega}+K_{\zeta}}\right),
\end{aligned}
$$

where $K_{\zeta}=\frac{1+K}{\zeta}$.

Using the series representation of the modified Bessel function of the first kind, i.e.,

$$
I_{v}(z)=\left(\frac{z}{2}\right)^{v} \sum_{n=0}^{\infty} \frac{\left(z^{2} / 4\right)^{n}}{n !(n+v) !}
$$

and noting that $I_{v}(z)=I_{-v}(z)$, the integral $C^{\prime}$ can be calculated as

$$
\begin{aligned}
& \dot{C}= \sum_{n=0}^{\infty} \sum_{k=1-N}^{N-1}\left(\frac{E_{m}}{\zeta}\right)^{k} \frac{\left(\overline{1} \sqrt{E_{m} \zeta}\right)^{2 n+|k|}}{n !(n+|k|) !} \int_{0}^{\infty} \beta^{2 n+|k|} \exp \left(-\left[\zeta+E_{m}\right] \bar{\beta}\right) f(\beta) d \beta \\
&= \sum_{n=0}^{\infty} \sum_{k=1-N}^{N-1}\left(\frac{E_{m}}{\zeta}\right)^{k} \frac{\left(\overline{1} \sqrt{E_{m} \zeta}\right)^{2 n+|k|}}{n !(n+|k|) !} \frac{(2 n+|k|+N-1) !}{(N-1) !} e^{-N K}\left(\frac{1}{2 \sigma_{h}^{2}}\right)^{N} \\
& \times\left(\overline{1}\left[\zeta+E_{m}\right]+\frac{1}{2 \sigma_{h}^{2}}\right)^{-(2 n+|k|+N)}{ }_{1} F_{1}\left(2 n+|k|+N ; N ; \frac{\lambda}{2\left(1+2 \bar{\sigma}_{h}^{2}\left(E_{m}+\zeta\right)\right)}\right) \\
&= \sum_{n=0}^{\infty} \sum_{k=1-N}^{N-1}\left(\frac{\sqrt{E_{m} \zeta}}{E_{m}+\zeta}\right)^{2 n+|k|}\left(\frac{E_{m}}{\zeta}\right)^{k} \frac{(2 n+|k|+N-1) !}{n !(n+|k|) !(N-1) !}\left(e^{-K} W_{m}\right)^{N} \frac{\bar{\Omega}^{2 n+|k|}}{\left(\bar{\Omega}+W_{m}\right)^{2 n+|k|+N}} \\
& \quad \times{ }_{1} F_{1}\left(2 n+|k|+N ; N ; \frac{N K W_{m}}{\bar{\Omega}+W_{m}}\right)
\end{aligned}
$$

where $W_{m} \triangleq \frac{1+K}{E_{m}+\zeta}$. It can be noticed that $\left(\frac{E_{m}}{\zeta}\right)^{n}$ in this CDF expression is converged only when $\frac{E_{m}}{\zeta}<1$ given that $n$ goes to infinity. Therefore, it will be used to calculate the error probability for $\operatorname{Pr}\left(\zeta \geq \frac{E_{m}+E_{m+1}}{2}\right)$. 
Similar to the case for $\zeta<E_{m}, \dot{B}$ and $\dot{C}$ for $\zeta>E_{m}$ can be asymptotically represented as

$$
\dot{B}^{\infty}=1-\left(\begin{array}{c}
2 N-1 \\
N
\end{array}\right)\left(\frac{K_{\zeta} e^{-K}}{\bar{\Omega}+K_{\zeta}} \frac{E_{m}}{\left(\frac{E_{m}}{\zeta}-1\right)^{2}}\right)^{N}
$$

and

$$
\begin{aligned}
\dot{C}^{\infty}= & \left(\frac{W_{m} e^{-K}}{\bar{\Omega}+W_{m}}\right)^{N} \sum_{k=1-N}^{N-1}\left(\frac{\sqrt{E_{m} \zeta}}{E_{m}+\zeta}\right)^{|k|}\left(\frac{E_{m}}{\zeta}\right)^{k} \sum_{n=0}^{\infty} \frac{(2 n+|k|+N-1) !}{n !(n+|k|) !(N-1) !}\left(\frac{\sqrt{E_{m} \zeta}}{E_{m}+\zeta}\right)^{2 n} \\
= & \left(\frac{W_{m} e^{-K}}{\bar{\Omega}+W_{m}}\right)^{N} \sum_{k=1-N}^{N-1}\left(\begin{array}{c}
N+|k|-1 \\
N-1
\end{array}\right)\left(\frac{\sqrt{E_{m} \zeta}}{E_{m}+\zeta}\right)^{|k|}\left(\frac{E_{m}}{\zeta}\right)^{k} \\
& \times{ }_{2} F_{1}\left(\frac{|k|+N}{2}, \frac{|k|+N+1}{2},|k|+1, \frac{4 E_{m} \zeta}{\left(E_{m}+\zeta\right)^{2}}\right) .
\end{aligned}
$$

C. The CDF for $E_{m}=0$ when $\zeta>E_{m}$

As the $\mathrm{CDF}$ in (55) is not valid for $E_{0}$, the $\mathrm{CDF} F_{\zeta}\left(\zeta \mid E_{0}\right)$ is derived separately. Towards this end, the conditional $\mathrm{CDF}$ given $\beta$ can be rewritten as

$$
\begin{aligned}
F_{\zeta}\left(\zeta \mid \beta, E_{0}\right) & =1-Q_{N}(0, \sqrt{2 \zeta \bar{\beta}}) \\
& =1-\frac{\Gamma(N, \zeta \bar{\beta})}{\Gamma(N)} \\
& =1-\exp (-\zeta \bar{\beta}) \sum_{k=0}^{N-1} \frac{(\zeta \bar{\beta})^{k}}{k !}
\end{aligned}
$$

where $\Gamma(\cdot, \cdot)$ is the complementary incomplete Gamma function. Thus, the unconditional CDF is computed as

$$
\begin{aligned}
F_{\zeta}\left(\zeta \mid E_{0}\right) & =1-\sum_{k=0}^{N-1} \frac{\bar{\zeta}^{k}}{k !} \int_{0}^{\infty} \beta^{k} \exp (-\zeta \bar{\beta}) f(\beta) d \beta \\
& =1-\sum_{k=0}^{N-1}\left(\begin{array}{c}
N+k-1 \\
k
\end{array}\right)\left(K_{\zeta} e^{-K}\right)^{N} \frac{(\bar{\Omega})^{k}}{\left(\bar{\Omega}+K_{\zeta}\right)^{k+N}}
\end{aligned}
$$

Asymptotically, this CDF can be represented as

$$
F_{\zeta}^{\infty}\left(\zeta \mid E_{0}\right)=1-\left(\begin{array}{c}
2 N-1 \\
N
\end{array}\right)\left(\frac{K_{\zeta} e^{-K}}{\bar{\Omega}+K_{\zeta}}\right)^{N} .
$$

By substituting the CDFs (51), (55) and (62) into (38), a closed-form expression can be obtained for the average SER by summation of infinite series. Moreover, by replacing the obtained CDFs with the asymptotic CDFs, the asymptotic average SER can be obtained in a closed-form. 
TABLE II

NORMALIZED TRUNCATION ERROR $T_{E}$ VERSUS THE SUMMATION LIMIT.

\begin{tabular}{|l||c|c|c|}
\hline $\mathcal{M}$ & $5 \mathrm{~dB}$ & $15 \mathrm{~dB}$ & $25 \mathrm{~dB}$ \\
\hline \hline 2 & $1.9 \times 10^{-1}$ & $2.7 \times 10^{-1}$ & $1.7 \times 10^{-3}$ \\
\hline 4 & $6.6 \times 10^{-3}$ & $1.1 \times 10^{-1}$ & $6.7 \times 10^{-4}$ \\
\hline 6 & $5.0 \times 10^{-3}$ & $4.0 \times 10^{-2}$ & $1.9 \times 10^{-4}$ \\
\hline 8 & $2.1 \times 10^{-4}$ & $1.2 \times 10^{-2}$ & $6.3 \times 10^{-6}$ \\
\hline 10 & $1.0 \times 10^{-3}$ & $5.0 \times 10^{-3}$ & $6.8 \times 10^{-5}$ \\
\hline 12 & $8.9 \times 10^{-4}$ & $4.0 \times 10^{-3}$ & $9.5 \times 10^{-5}$ \\
\hline 14 & $9.0 \times 10^{-4}$ & $4.2 \times 10^{-3}$ & $1.0 \times 10^{-4}$ \\
\hline 16 & $9.0 \times 10^{-4}$ & $4.3 \times 10^{-3}$ & $1.0 \times 10^{-4}$ \\
\hline 18 & $9.0 \times 10^{-4}$ & $4.4 \times 10^{-3}$ & $1.0 \times 10^{-4}$ \\
\hline 20 & $9.0 \times 10^{-4}$ & $4.4 \times 10^{-3}$ & $1.1 \times 10^{-4}$ \\
\hline
\end{tabular}

\section{NumericAl Results}

This section presents the analytical and simulation SER of MASK modulation with coherent, noncoherent and amplitude-coherent detection in flat Rician fading channels. The SER for AC detector is presented for the optimum, suboptimum, and the heuristic detectors, using single and multiple antennas as well as different modulation orders. The Monte Carlo simulation results are obtained by generating $10^{7}$ realizations and the average SNR is defined as $S N R=\frac{\Omega \bar{E}_{S}}{2 \sigma_{n}^{2}}$, where $\bar{E}_{S}=\frac{1}{M} \sum_{m=0}^{M-1} E_{m}$ is the average symbol energy. In the analytical results, the summations with infinite limits are truncated to 20 terms, unless it is specified otherwise. For the results included in this section $\bar{E}_{S}$ and $\Omega$ are normalized to 1 . The figures' legends are using the following abbreviations, simulation (Sim.), analytical (Anal.), coherent detector (Coh.), noncoherent detector (NC), near-optimum AC (AC-NO), suboptimum AC (AC-SO), AC heuristic (AC-H), and asymptotic (Asymp.).

Table II depicts the accuracy of the truncated infinite summation, where the normalized truncation error $T_{E}$ is calculated as the normalized difference between the analytical and simulation results, $T_{E}=\mid P_{\mathrm{e}}($ Sim. $)-P_{\mathrm{e}}($ Anal. $) \mid / P_{\mathrm{e}}($ Sim. $)$. The results in Table II are obtained for various summation limits using different SNRs, while $M=2, N=1$ and $K=4$. The results in the Table show that the analytical and simulation results generally converge when the summation limit is equal or more than 6 .

Fig. 1 shows the analytical and simulated conditional PDF $f\left(\zeta \mid E_{m}\right)[41]$ where $M=4, K=10$ and $S N R=27 \mathrm{~dB}$. As can be noted from the figure, the overlap between the conditional PDF 


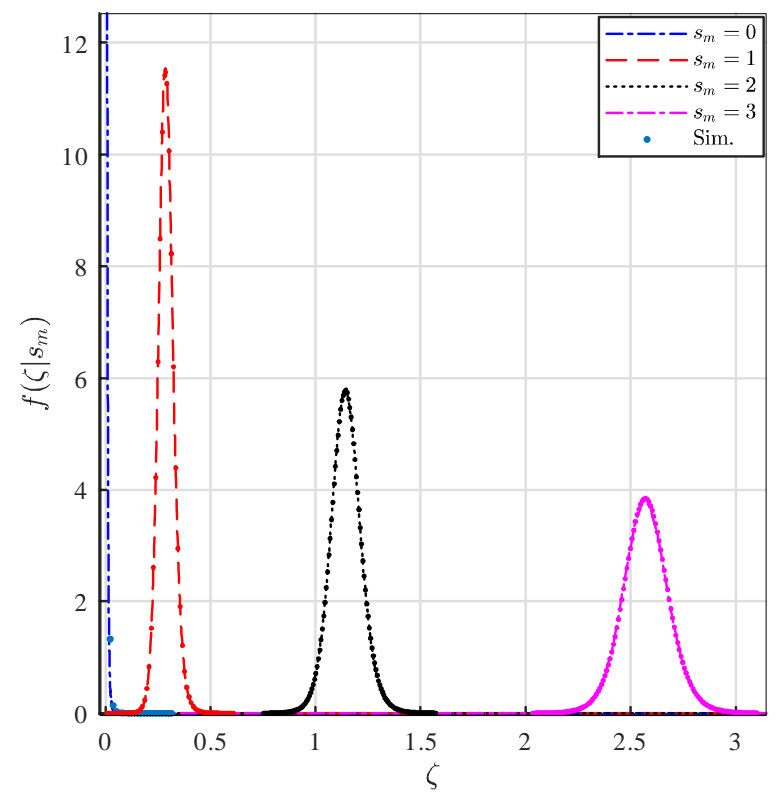

Fig. 1. Analytical and simulated conditional PDF $f\left(\zeta \mid E_{m}\right)$ for $M=4, K=10$ and $S N R=27 \mathrm{~dB}$.

for different $E_{m}$ values at such high SNR is negligible, and thus, the transmitted signal can be recovered reliably using the thresholds described in (34) for the heuristic AC detector. However, because the conditional PDFs are not identical and not equally spaced, the probability of error will depend on the transmitted symbol. Consequently, the amplitudes of the transmitted symbols can be optimized to minimize the BER. Nevertheless, the improvement that would be gained is generally limited as reported for the Rayleigh fading case [32].

Figs. 2 and 3 compare the SER of the coherent and heuristic AC detectors using $M=2$ and 4 , respectively. The figures also show the SER when the number of receiving antennas $N=1,2$, and 4, the Rician factor for both figures is $K=4$. The results presented in both figures show that the simulated SER perfectly matches the analytical SER for all the considered $M, N$ and SNR values. Comparing the coherent and $\mathrm{AC}$ heuristic detectors for $M=2$ in Fig. 2 shows that the coherent detector outperforms the AC detector by about $3 \mathrm{~dB}$ at $P_{\mathrm{e}}=5 \times 10^{-5}$. For $M=4$ in Fig. 3, the difference between the coherent and AC Heuristic becomes about 1.4, 1.7 and $2.0 \mathrm{~dB}$ for $N=1,2$, and 3 , respectively, at $P_{\mathrm{e}}=5 \times 10^{-5}$.

Fig. 4 illustrates the impact of the Rician factor $K$ on the SER for $M=2,4$, and $N=1$. The results for $K=0$ are considered as the worst case scenario where the channel becomes Rayleigh. As can be depicted from the figure, the SER of the AC detector improves substantially 


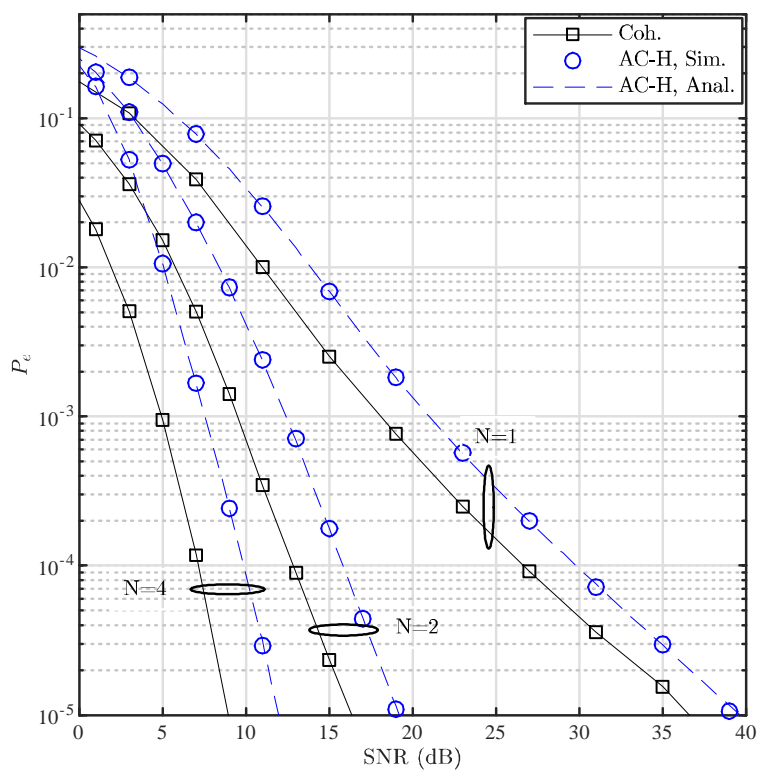

Fig. 2. Analytical and simulated SER of the AC heuristic (AC-H) and coherent detectors using $N=1,2,4, M=2$, and $K=4$.

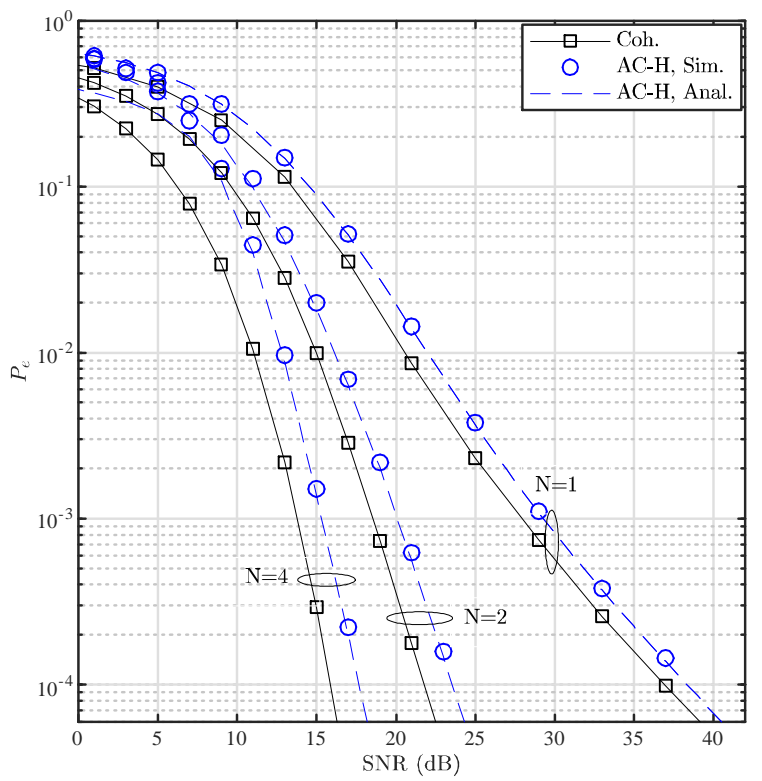

Fig. 3. Analytical and simulated SER of the AC heuristic (AC-H) and coherent detectors using $N=1,2,4, M=4$, and $K=4$. 


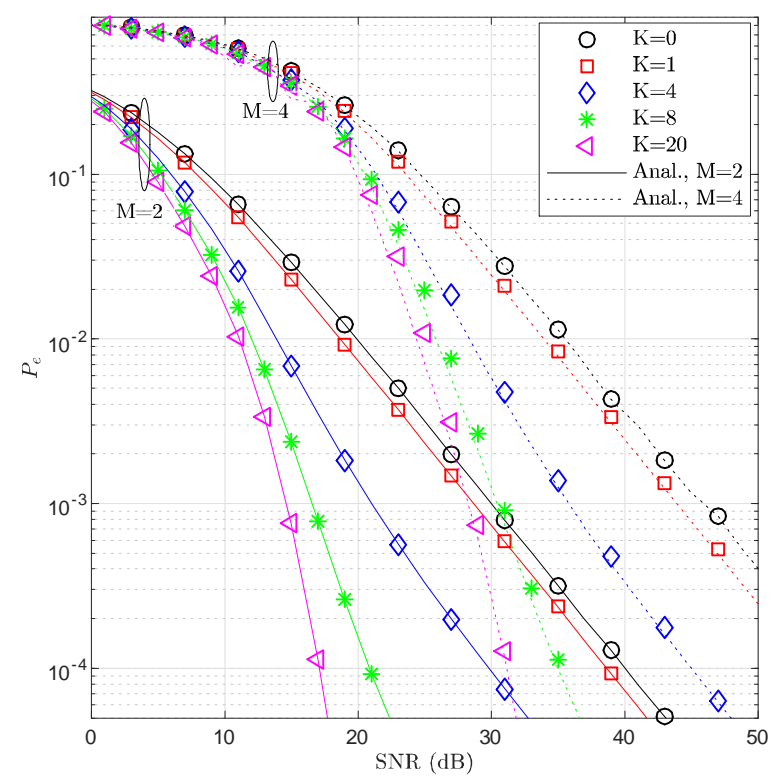

Fig. 4. Analytical and simulated SER of the AC heuristic (AC-H) detector using $M=2$ and 4 for different values of $K, N=1$.

by increasing $K$. For example, the SER improvement gained by increasing $K$ from 0 to 20 is about $20 \mathrm{~dB}$ at $P_{e}=4 \times 10^{-4}$ for both modulation orders.

Figs. 5 and 6 compare the SER of the near-optimum, suboptimum and heuristic AC detectors for $M=2,4, N=1,2$ and $K=4$. As shown in both figures, the heuristic detector outperforms the suboptimum for all cases. For $M=2$, the difference is about 3.5 and $3.7 \mathrm{~dB}$ for $N=1$ and 2, respectively. For $M=4$, the difference is about 2.5 and $3 \mathrm{~dB}$ for $N=1$ and 2, respectively. As expected, the near-optimum detector outperforms the heuristic for $S N R \lesssim 21 \mathrm{~dB}$ for $M=2$ and $N=1$, which corresponds to low and moderate SNRs. For $N=2$, the system SNR is generally much smaller than the $N=1$ case, and hence, the near-optimum detector outperforms the heuristic. The SER behavior for $M=4$ given in Fig. 6 is generally similar to the $M=2$ case, except that the cross-over point is shifted to $S N R \approx 29 \mathrm{~dB}$. Consequently, the heuristic detector offers the best compromise between SER and computational complexity as compared to the suboptimum and near-optimum detectors.

Fig. 7 presents the SER using the optimum noncoherent, heuristic AC and PC detectors for that $M=2,4, N=1$, and $K=4$. As can be noted from the figure, the optimum noncoherent and heuristic PC detectors have roughly equivalent SER for $M=4$, but the PC slightly outperforms the noncoherent for the $M=2$ case. Moreover, both of detectors outperform the heuristic AC detector 


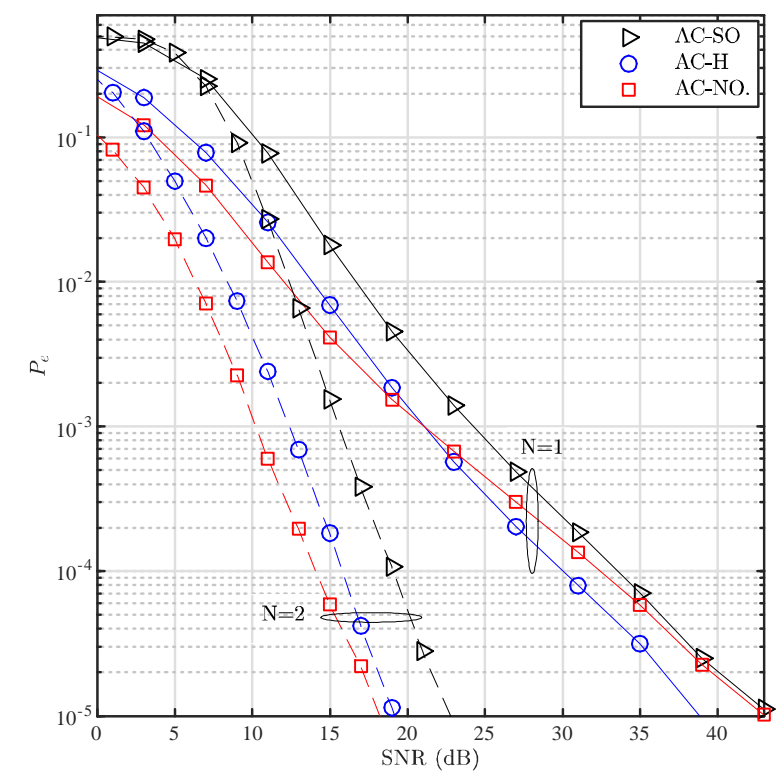

Fig. 5. SER for of the near optimum (AC-NO), suboptimum (AC-SO), and heuristic AC (AC-H) detectors using $N=1,2$, $M=2$, and $K=4$.

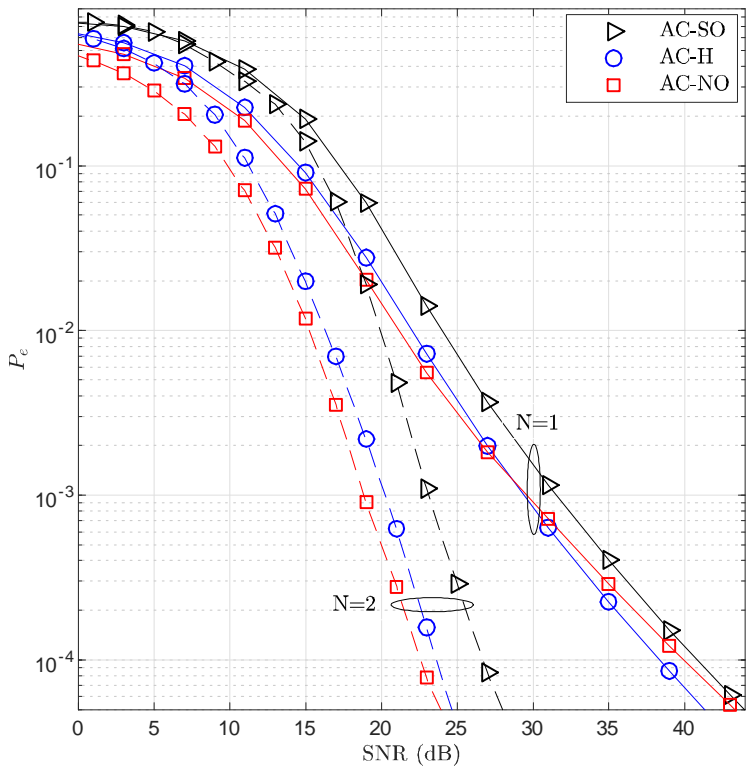

Fig. 6. SER for of the near optimum (AC-NO), suboptimum (AC-SO) and heuristic AC (AC-H) detectors using $N=1,2$, $M=4$, and $K=4$. 


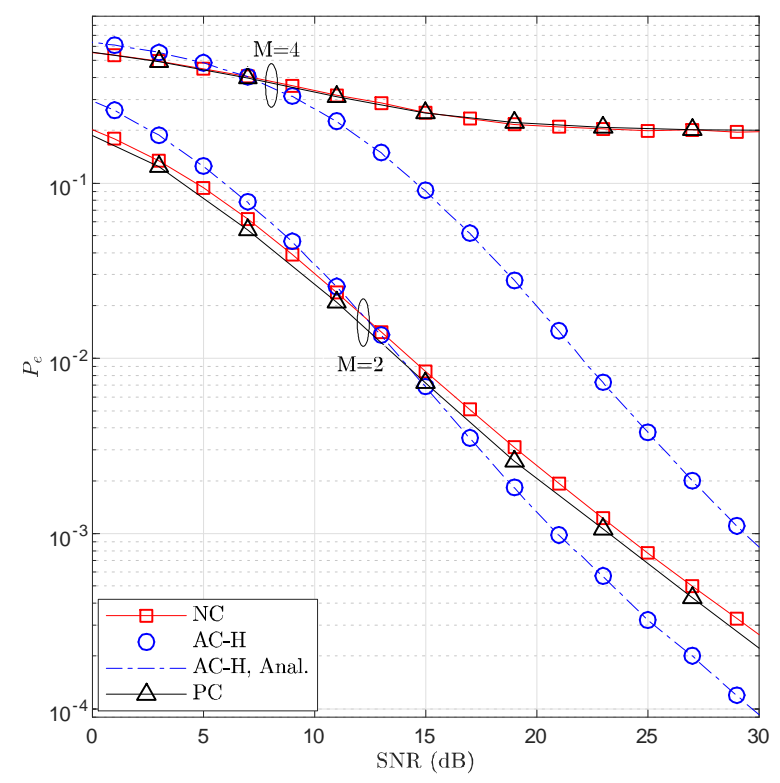

Fig. 7. The SER for the noncoherent (NC) and heuristic (AC-H) detectors using $M=2,4, N=1$ and $K=4$.

for SNRs less than 11 and $5 \mathrm{~dB}$, for $M=2$ and 4 respectively. Nevertheless, the noncoherent and PC detectors SER deteriorates severely for $M>2$ where an error floor is observed at $S E R \sim 2 \times 10^{-1}$. Moreover, it is worth noting that the optimum noncoherent detector requires prior knowledge of the channel statistical information, i.e., $\sigma_{n}^{2}, \sigma_{h}^{2}$ and $\mu_{h}$.

Figs. 8 and 9 show the effect of the phase noise on both the heuristic and coherent detectors for the cases of $M=2$ and 4 , respectively. The parameters for the two figures are $N=1$ and $K=4$, and the phase noise is modeled as a Tikhonov random variable with variance in degrees $\sigma_{\phi}^{2}=[0,3,5,7,10]$ [44], [45]. In both figures, the SER of the AC detector is represented by a single curve because it is immune to phase noise. The results in Figs. 8 and 9 show clearly the advantage of the AC detection in the presence of phase noise, particularly at high SNRs, where the coherent detector exhibits SER error floors with high values. As expected, the SER degradation is proportional to the modulation order. For example, the error floor for $M=4$ results for values of $\sigma_{\phi}^{2}$ as low as $3^{\circ}$.

Fig. 10 compares the asymptotic and analytical SERs using $M=2, K=4, N=1,2$ and 3. As can be noted from the figure, the asymptotic SER provides accurate results for the SER at high SNRs, and thus, it can be useful to estimate the SER of the AC heuristic detector. 


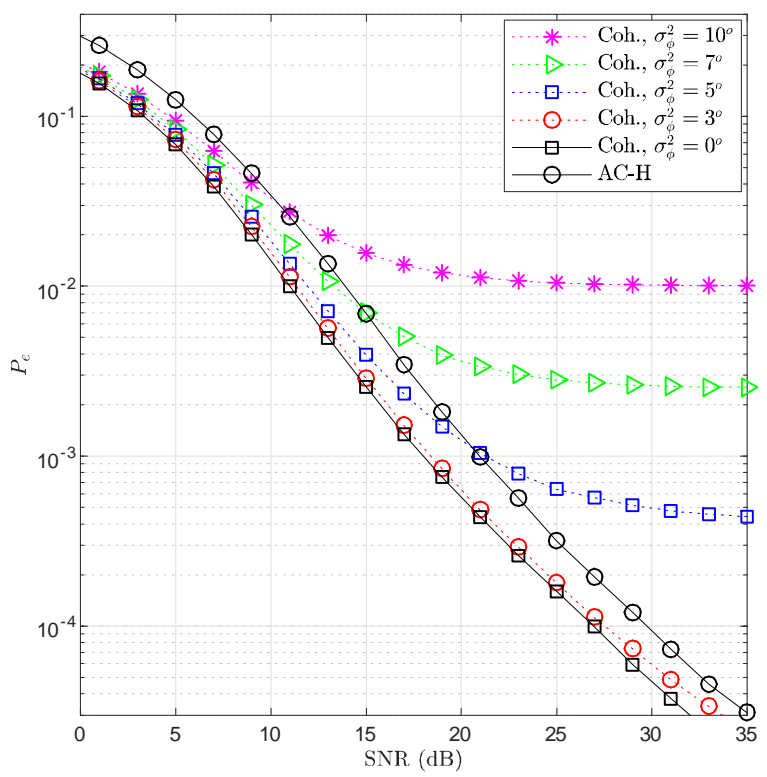

Fig. 8. The effect of the phase noise on the heuristic AC (AC-H) and coherent detectors for $N=1$, where $M=2, K=4$.

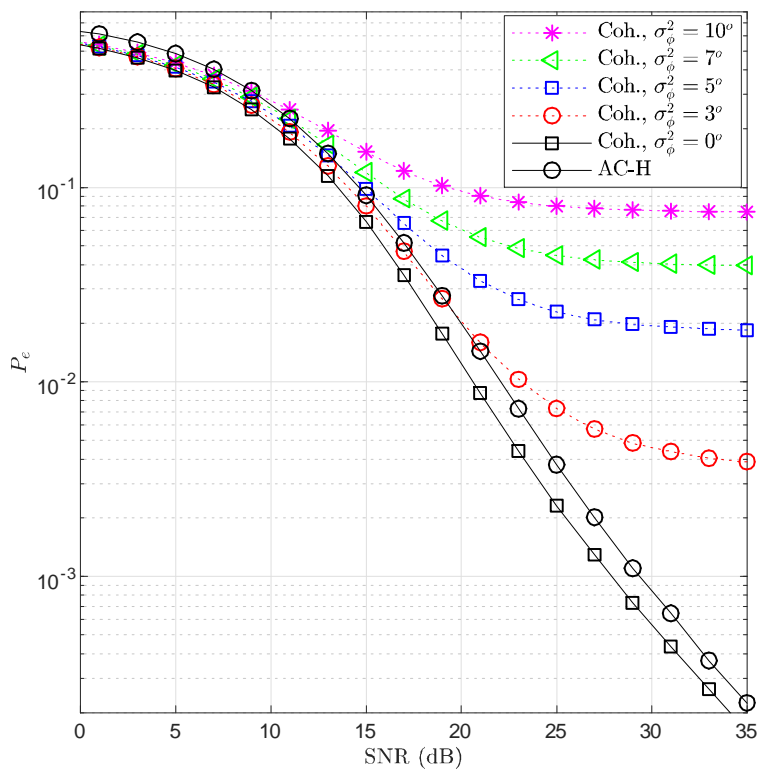

Fig. 9. The effect of the phase noise on the heuristic $\mathrm{AC}(\mathrm{AC}-\mathrm{H})$ and coherent detectors for $N=1$, where $M=4, K=4$. 


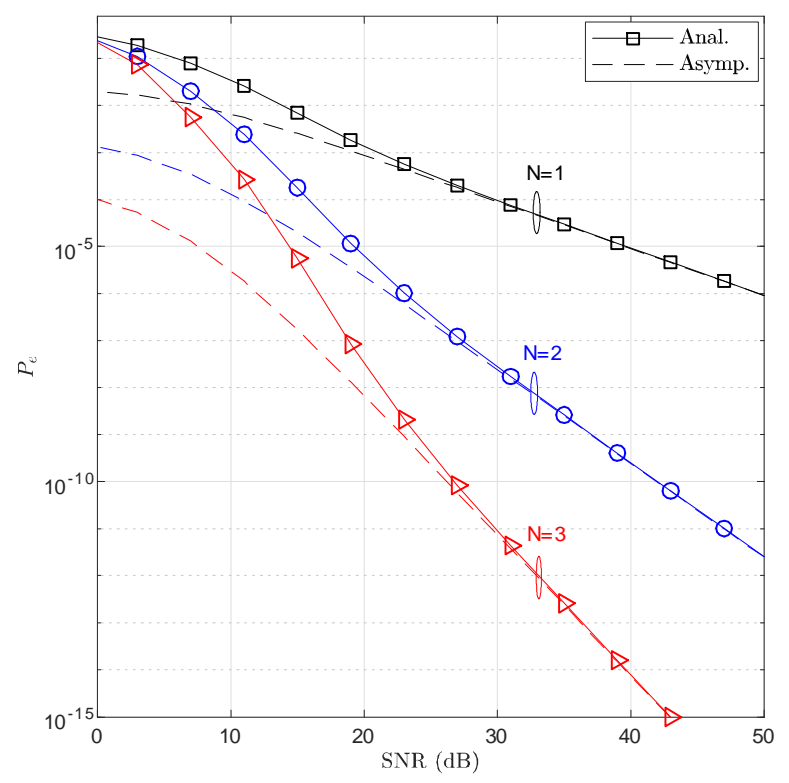

Fig. 10. Asymptotic SER using $M=2, K=4$, and $N=1,2$ and 3 .

\section{CONCLUSION AND Future WORK}

In this paper, the SER performance of MASK modulation with amplitude-coherent detection has been considered over flat Rician fading channels with receiver diversity. A closed form analytical expression was derived for the SER of the heuristic detector, and then, a simple formula was derived for asymptotic SER at high SNRs. Moreover, the optimum, near-optimum and suboptimum amplitude-coherent detectors were derived for the Rician fading channel, and their SER was compared with the heuristic amplitude-coherent, coherent, phase-coherent and noncoherent detectors for various modulation orders and number of receiving antennas. The obtained analytical and simulation results confirm that the amplitude-coherent detection offers SER performance that is comparable to the coherent detection, but without the need for a prior knowledge of the channel phase. Consequently, the computational complexity of the amplitudecoherent detector is much less than the coherent detector. The simulation results in the presence of phase noise corroborated that advantage of the amplitude-coherent detector over the coherent detector in the presence of phase noise. 


\section{APPENDIX I: EVALUATING THE INTEGRAL $\mathcal{I}_{\theta}$}

The integral $\mathcal{I}_{\theta}$ given in (23) can be written as

$$
\mathcal{I}_{\theta}=\frac{1}{2 \pi} \int_{-\pi}^{\pi} \exp \left(\mathcal{K} \cos (\theta-\phi)+2 \bar{\alpha} s_{m}|r| \cos \left(\theta-\theta_{\mathbf{r}}\right)\right) d \theta .
$$

Applying the trigonometric identity $\cos (\theta-\phi)=\cos \theta \cos \phi+\sin \theta \sin \phi$ yields

$$
\begin{aligned}
& \mathcal{I}_{\theta}= \frac{1}{2 \pi} \int_{-\pi}^{\pi} \exp [\mathcal{K}(\cos (\theta) \cos (\phi)+\sin (\theta) \sin (\phi))] \\
& \quad \times \exp \left[2 \bar{\alpha} s_{m}|r|\left(\cos (\theta) \cos \left(\theta_{\mathrm{r}}\right)+\sin (\theta) \sin \left(\theta_{\mathrm{r}}\right)\right)\right] d \theta \\
&=\frac{1}{2 \pi} \int_{-\pi}^{\pi} \underbrace{\exp \left(\left(A+B \alpha s_{m}|r|\right) \cos (\theta)+\left(C+D \alpha s_{m}|r|\right) \sin (\theta)\right)}_{g(\theta)} d \theta
\end{aligned}
$$

where $A=\mathcal{K} \cos (\phi), B=\overline{2} \cos \left(\theta_{\mathrm{r}}\right), C=\mathcal{K} \sin (\phi)$ and $D=\overline{2} \sin \left(\theta_{\mathrm{r}}\right)$. However, because $g(\theta)$ is periodic, then the integral can be written as

$$
\begin{aligned}
\int_{-\pi}^{\pi} g(\theta) d \theta & =\int_{-\pi}^{\pi} g(\theta+\varphi) d \theta \\
& =2 \int_{0}^{\pi} g(\theta+\varphi) d \theta
\end{aligned}
$$

where $\varphi=\arg \max _{\tilde{\theta}} g(\theta)$, which can be derived as

$$
\begin{aligned}
\frac{d g(\theta)}{d \theta}=\left[-\left(A+B \alpha s_{m}|r|\right) \sin \theta\right. & \left.+\left(C+D \alpha s_{m}|r|\right) \cos \theta\right] \\
& \times \exp \left(\left(A+B \alpha s_{m}|r|\right) \cos \theta+\left(C+D \alpha s_{m}|r|\right) \sin \theta\right) .
\end{aligned}
$$

To find $\varphi \in(-\pi, \pi)$, the unique root of $\frac{d g(\theta)}{d \theta}=0$ is calculated,

$$
\varphi=\tan ^{-1}\left(\frac{C+D \alpha s_{m}|r|}{A+B \alpha s_{m}|r|}\right) \text {. }
$$

Therefore,

$$
g(\theta+\varphi)=\exp \left(\left(A+B \alpha s_{m}|r|\right) \cos (\theta+\varphi)+\left(C+D \alpha s_{m}|r|\right) \sin (\theta+\varphi)\right)
$$

Therefore, the integral $\mathcal{I}_{\theta}$ given in (64) can be rewritten as as

$$
\mathcal{I}_{\theta}=\frac{1}{\pi} \int_{0}^{\pi} g(\theta+\varphi) d \theta
$$


Evaluating the integral $\mathcal{I}_{\theta}$ by substituting $y=\cos (\theta)$ yields

$$
\begin{aligned}
\mathcal{I}_{\theta}=\frac{1}{\pi} \int_{-1}^{1} \frac{1}{\sqrt{1-y^{2}}} \exp \left\{\left(A+B \alpha s_{m}|r|\right) \cos (\varphi\right. & \left.+\cos ^{-1}(y)\right) \\
& \left.+\left(C+D \alpha s_{m}|r|\right) \sin \left(\varphi+\cos ^{-1}(y)\right)\right\} d y
\end{aligned}
$$

which can be efficiently solved using the Gauss-Chebyshev quadrature rules [46, Eq. 25.4.38],

$$
\mathcal{I}_{\theta}=\frac{1}{L} \sum_{l=1}^{n} \exp \left[\left(A+B \alpha s_{m}|r|\right) f_{\mathrm{c}}\left(y_{l}\right)+\left(C+D \alpha s_{m}|r|\right) f_{\mathrm{s}}\left(y_{l}\right)\right],
$$

where

$$
\begin{gathered}
f_{\mathrm{c}}\left(y_{l}\right)=\cos \left(\varphi+\cos ^{-1}\left(y_{l}\right)\right)=\cos \left(\varphi+\frac{2 l-1}{2 L} \pi\right) \\
f_{\mathrm{s}}\left(y_{l}\right)=\sin \left(\varphi+\cos ^{-1}\left(y_{l}\right)\right)=\sin \left(\varphi+\frac{2 l-1}{2 L} \pi\right) \\
y_{l}=\cos \left(\frac{2 l-1}{2 L} \pi\right) .
\end{gathered}
$$

\section{REFERENCES}

[1] A. Al-Dweik and Y. Iraqi, "Error probability analysis and applications of amplitude-coherent detection in flat Rayleigh fading channels," IEEE Trans. Commun., vol. 64, no. 5, pp. 2235-2244, May 2016.

[2] Digital Video Broadcasting (DVB); Framing Structure, Channel Coding and Modulation for Digital Terrestrial Television, ETSI Standard EN 300744 v1.6.1, 2008.

[3] IEEE Standard for Local and Metropolitan Area Networks Part 16: Air Interface for Broadband Wireless Access Systems Amendment 3: Advanced Air Interface, IEEE Standard 802.16m, 2011.

[4] LTE; Evolved Universal Terrestrial Radio Access (E-UTRA), LTE Physical Layer, 3GPP TS 36.300, 2011.

[5] F. Yang and L. Yang, "Low-complexity noncoherent fusion rules for wireless sensor networks monitoring multiple events," IEEE Trans. Aerosp. Electron. Syst., vol. 50, no. 3, pp. 2343-2353, Jul. 2014.

[6] S. Bi, C. K. Ho, and R. Zhang, "Wireless powered communication: Opportunities and challenges," IEEE Commun. Mag., vol. 53, no. 4, pp. 117-125, Apr. 2015.

[7] M. Al-Jarrah, A. Al-Dweik, E. Alsusa, and E. Damiani, "RFID reader localization using hard decisions with error concealment," IEEE Sensors J, IEEE Xplore early access, DOI: 10.1109/JSEN.2019.2914914.

[8] Z. Zhao, Z. Zhang, J. Tan, Y. Liu, and J. Liu, "200 Gb/s FSO WDM communication system empowered by multiwavelength directly modulated TOSA for 5G wireless networks," IEEE Photon. J., vol. 10, no. 4, pp. 1-8, Aug. 2018.

[9] J. Rodríguez, D. Lamar, P. Miaja, and J. Sebastián, "Reproducing single-carrier digital modulation schemes for VLC by controlling the first switching harmonic of the DC-DC power converter output voltage ripple," IEEE Trans. Power Electron., vol. 33, no. 9, pp. 7994-8010, Sep. 2018.

[10] A. Belmonte and J. M. Kahn, "Performance of synchronous optical receivers using atmospheric compensation techniques," Opt. Express, vol. 16, no. 18, pp. 14151-14162, Sep. 2008. 
[11] A. Touati, A. Abdaoui, F. Touati, M. Uysal, and A. Bouallegue, "On the effects of combined atmospheric fading and misalignment on the hybrid FSO/RF transmission," IEEE/OSA J. Opt. Commun. Netw., vol. 8, no. 10, pp. 715-725, Oct. 2016.

[12] P. Trinh, T. Thang, and A. Pham, "Mixed mmwave RF/FSO relaying systems over generalized fading channels with pointing errors," IEEE Photon. J., vol. 9, no. 1, pp. 1-14, Feb. 2017.

[13] M. A. Khalighi and M. Uysal, "Survey on free space optical communication: A communication theory perspective," IEEE Commun. Surveys Tuts., vol. 16, no. 4, pp. 2231-2258, 2014.

[14] D. Tsonev, S. Sinanovic and H. Haas, "Complete modeling of nonlinear distortion in OFDM-based optical wireless communication,” J. Lightw. Technol., vol. 31, no. 18, pp. 3064-3076, Sep. 2013.

[15] H. Lu, C. Li, C. Ho, M. Cheng, X. Lin, Z. Yang, and H. Chen, "64 Gb/s PAM4 VCSEL-based FSO link," Opt. Express, vol. 25, no. 5, pp. 5749-5757, Mar. 2017.

[16] J. Armstrong and B. Schmidt, "Comparison of asymmetrically clipped optical OFDM and DC-biased optical OFDM in AWGN,” IEEE Commun. Lett., vol. 12, no. 5, pp. 343-345, May 2008.

[17] M. Zhang and Z. Zhang, "An optimum DC-biasing for DCO-OFDM system," IEEE Commun. Lett., vol. 18, no. 8, pp. 1351-1354, Aug. 2014.

[18] N. Letzepis and A. Fabregas, "Outage probability of the freespace optical channel with doubly stochastic scintillation," IEEE Trans. Commun., vol. 57, no. 10, pp. 2899-2902, Oct. 2009.

[19] S. Aghajanzadeh and M. Uysal, "Diversity-multiplexing trade-off in coherent free-space optical systems with multiple receivers," IEEE/OSA J. of Opt. Commun. Netw., vol. 2, no. 12, pp. 1087-1094, Dec. 2010.

[20] K. Jung, S. S. Nam, Y. Ko and M.-S Alouini, "BER performance of FSO links over unified channel model for pointing error models," IEEE Int. Conf. Commun., (ICC Workshops), Kansas City, USA, May 2018, pp. 1-6.

[21] F. Yang, J. Cheng and T. A. Tsiftsis, "Free-space optical communications with generalized pointing errors," IEEE Int. Conf. Commun. (ICC), Budapest, 2013, pp. 3943-3947.

[22] I. S. Ansari, M.-S Alouini and J. Cheng, "On the capacity of FSO links under Lognormal and Rician-Lognormal turbulences," IEEE 80th Veh. Technol. Conf. (VTC Fall), Vancouver, BC, 2014, pp. 1-6.

[23] D. Yue, Y. Zhang, and Y. Jia, "Beamforming based on specular component for massive MIMO systems in Ricean fading," IEEE Wireless Commun. Lett., vol. 4, no. 2, pp. 197-200, Apr. 2015.

[24] S. Jin, D. Yue, and H. Nguyen, "Equal-gain transmission in massive MIMO systems under Ricean fading," IEEE Trans. Veh. Technol., vol. 67, no. 10, pp. 9656-9668, Oct. 2018.

[25] H. Tataria, P. Smith, L. Greenstein, and P. Dmochowski, "Zero-forcing precoding performance in multiuser MIMO systems with heterogeneous Ricean fading," IEEE Wireless Commun. Lett., vol. 6, no. 1, pp. 74-77, Feb. 2017.

[26] J. Nyarko, J. Xie, R. Yao, Y. Wang, and L. Wang, "Accurate approximation of ZF massive MIMO channel rate with a finite antenna over Ricean fading channel," IEEE Access, vol. 6, pp. 65803-65812, 2018.

[27] X. Sun, K. Xu, W. Ma, Y. Xu, X. Xia, and D. Zhang, "Multi-pair two-way massive MIMO AF full-duplex relaying with imperfect CSI over Ricean fading channels,” IEEE Access, vol. 4, pp. 4933-4945, 2016.

[28] R. Sun and D. Matolak, "Air-ground channel characterization for unmanned aircraft systems Part II: Hilly and mountainous settings," IEEE Trans. Veh. Technol., vol. 66, no. 3, pp. 1913-1925, Mar. 2017.

[29] D. Matolak and R. Sun, "Air-ground channel characterization for unmanned aircraft systems Part III: The suburban and near-urban environments," IEEE Trans. Veh. Technol., vol. 66, no. 8, pp. 6607-6618, Aug. 2017. 
[30] ${ }^{\left[C_{1}\right]}$ Y. Gao, Y. Chen, and A. Bekkali, "Performance of passive UHF RFID in cascaded correlated generalized Rician fading," IEEE Commun. Lett., vol. 20, no. 4, pp. 660-663, Apr. 2016.

[31] ${ }^{\left[C_{1}\right]}$ C. Oestges, D. Vanhoenacker-Janvier, and B. Clerckx, "Channel characterization of indoor wireless personal area networks," IEEE Trans. Antennas Propag., vol. 54, no. 11, pp. 3143-3150, Nov. 2006.

[32] L. Bariah, A. Al-Dweik, S. Muhaidat, Y. Iraqi, and M. Al-Mualla, "Performance analysis of semi-coherent OFDM systems with imperfect channel estimates,” IEEE Trans. Veh. Technol., vol. 677, no. 11, pp. 10773-10787, Nov. 2018.

[33] L. Bariah, A. Al-Dweik and S. Muhaidat, "Blind channel estimation technique for OFDM systems over time varying channels," IEEE 87th Vehic. Technol. Conf. (VTC Spring), Porto, 2018, pp. 1-7.

[34] R. K. Mallik, R. D. Murch and Y. Li, "Channel magnitude based energy detection with receive diversity for multi-level amplitude-shift keying in Rayleigh fading," IEEE Trans. Commun., vol. 65, no. 7, pp. 3079-3094, Jul. 2017.

[35] D. Matolak and R. Sun, "Air-ground channel characterization for unmanned aircraft systems-Part III: The suburban and near-urban environments," IEEE Trans. Vehic. Technol., vol. 66, pp. 6607-6618, Aug. 2017.

[36] R. Dana, "Statistics of sampled Rician fading," DNA-TR-92-98 Tech. Rep., Feb. 1993.

[37] M. K. Simon and M.-S. Alouini, Digital Communication over Fading Channels: A Unified Approach to Performance Analysis, 2nd ed. NY: A Wiley-Interscience Publication, 2000.

[38] A. Saci, A. Al-Dweik, A. Shami and Y. Iraqi, "One-shot blind channel estimation for OFDM systems over frequency-selective fading channels," IEEE Trans. Commun., vol. 65, no. 12, pp. 5445-5458, Dec. 2017.

[39] I. B. G. Pôrto and M. D. Yacoub, "On the phase statistics of the $\kappa$ - $\mu$ process," IEEE Trans. Wireless Commun., vol. 15, no. 7, pp. 4732-4744, July 2016.

[40] J. Proakis and M. Salehi, Digital Communications, 5th ed. UK: Ashford Colour Press, 2014.

[41] M. Al-Jarrah, K. Park, A. Al-Dweik, and M. S. Alouini, "Error rate analysis of amplitude-coherent detection over Rician fading channels with receiver diversity," arXiv preprint arXiv:1902.01812. 2019 Feb. 5.

[42] S. András, Á. Baricz, and Y. Sun, “The generalized Marcum Q-function: An orthogonal polynomial approach,” Acta Univ. Sapientiae Math., vol. 3, no. 1, pp. 60-76, 2011.

[43] A. P. Prudnikov, Yu. A. Brychkov, and O. I. Marichev, Integrals and Series, vol. 2 Special Functions. Gordon and Breach Science Publishers, UK, 3rd edition, 1986.

[44] F. Kayhan and G. Montorsi, "Constellation design for memoryless phase noise channels," IEEE Trans. Wireless Commun., vol. 13, no. 5, pp. 2874-2883, May 2014.

[45] R. Krishnan, A. Graell, T. Eriksson, and G. Colavolpe, "Constellation optimization in the presence of strong phase noise," IEEE Trans. Commun., vol. 61, no. 12, pp. 5056-5066, Dec. 2013.

[46] M. Abramowitz and I. Stegun, I A, Handbook of Mathematical Functions, 10th printing with corrections, 1972. 